\title{
PRIMA DI GOMORRA. LA CAMORRA ANNI OTTANTA SECONDO PASQUALE GALASSO
}

A cura di Ciro Dovizio

Title: Before Gomorrah. The camorra in the Eighties in the view of Pasquale Galasso

\begin{abstract}
This article examines an extract of the hearing of the pentito Pasquale Galasso before the antimafia Parliamentary Committee on 13 July 1993, the text of which is reported hereafter. It examines the salient points of the testimony, focusing on the Nuova camorra organizzata (NCO), a camorristic group founded by Raffaele Cutolo at the end of the 1970s, on some of its original profiles and on the role it played in the Campania context. Galasso's point of view on the gang wars of those years and particularly on the disproportionate use of violence is also discussed.
\end{abstract}

Key words: Galasso, Camorra, Cutolo, Nuova camorra organizzata, violence

Si prende qui in esame uno stralcio dell'audizione del pentito di camorra Pasquale Galasso davanti alla Commissione parlamentare antimafia del 13 luglio 1993, il cui testo è riportato di seguito. L'articolo esamina i punti salienti della testimonianza, focalizzando l'attenzione sulla Nuova camorra organizzata (Nco), gruppo camorristico fondato da Raffaele Cutolo alla fine degli anni settanta, su alcuni suoi profili di originalità e sul ruolo da essa svolto nel contesto campano. Si discute inoltre il punto di vista di Galasso sulle guerre per bande di quegli anni e particolarmente sull'uso sproporzionato della violenza.

Parole chiave: Galasso, Camorra, Cutolo, Nuova camorra organizzata, violenza 
Nella sezione "Storia e memoria" di questo numero la rivista propone un ampio stralcio dell'audizione di Pasquale Galasso alla Commissione parlamentare antimafia (presidente Luciano Violante) del 13 luglio 1993. Galasso (tuttora vivente e libero) è stato top-leader del gruppo camorristico Alfieri, egemone a metà anni ottanta nell'area nolano-vesuviana e in quella nocerino-sarnese. Nacque nel 1955 a Poggiomarino, provincia di Napoli, da una famiglia assai facoltosa: il padre Sabato, ex proprietario terriero, aveva fatto fortuna vendendo autocarri Fiat, sicché a Pasquale non erano mancati benessere e ogni sorta di lusso (auto da corsa, abiti firmati, locali alla moda). Nel 1975, mentre studiava medicina, reagì a un tentativo di sequestro uccidendo due dei rapitori. Fu dunque condannato a un anno di reclusione per eccesso di legittima difesa. Nel carcere di Poggioreale entrò in contatto con numerosi capi-camorra: dall'astro nascente Raffaele Cutolo, accanto alla cui cella trascorse parte della detenzione, ai fratelli Michele e Salvatore Zaza, dai Nuvoletta agli Sciorio, ai Maisto, i quali tentarono più volte di reclutarlo, ma senza successo. Stando alla sua stessa testimonianza, infatti, l'affiliazione - senza rituale iniziatico - sarebbe venuta al rientro, e cioè a Poggiomarino: qui, per fronteggiare gli estortori del padre, si sarebbe legato a Salvatore Alfieri, boss della zona, e al fratello Carmine, capo dell'omonimo clan nolano. Nel gruppo Alfieri, Galasso avrebbe ricoperto sin dall'inizio ruoli di responsabilità, macchiandosi di decine di delitti, capitanando gruppi di fuoco, gestendo risorse e investimenti, per divenire collaboratore di giustizia nei primi anni novanta.

Come tutte le rivelazioni dall'interno, anche quelle di Galasso appaiono un po' apologetiche e un po' depistanti: il loro valore, però, già appurato in sede giudiziaria, resta a tutt'oggi indiscutibile. Esse conducono a un tempo non più nostro e, per molti aspetti, remoto: parliamo di quegli anni ottanta che, celebrati come canto del cigno della modernità italiana, tra secolarizzazione, sviluppo economico, tv commerciali, allargamento dei consumi, nuovi stili di vita, videro anche l'ascesa vertiginosa delle mafie, il loro ostentato protagonismo, la loro espansione dalle aree di più tradizionale insediamento al resto del paese. Chiamando in causa camorristi, mafiosi, esponenti politici, professionisti, agenti segreti, evocando delitti e intrecci politico-affaristico-criminali, Galasso ci racconta un pezzo di storia d'Italia non secondario ma a lungo, e forse ancor oggi, trascurato. 
Tra gli argomenti dell'audizione c'è naturalmente Raffaele Cutolo, originario di Ottaviano, fondatore nei secondi anni settanta di un originalissimo gruppo criminale, la Nuova camorra organizzata (Nco). Incredibilmente, Cutolo costruì e alimentò questa sua creatura dal carcere incontrando - ha spiegato Isaia Sales - la devianza giovanile, reclutandola dove viveva collettivamente (periferie urbane e carcere), compattandola, dandole "una bandiera e un credo", nonché un testo "sacro", il suo libro Poesie e pensieri. Egli presentò sé stesso come un moderno Robin Hood, portatore di giustizia in un mondo iniquo, benefattore di un popolo abbandonato, prostrato dalla miseria e dalla disoccupazione. Con lui si ebbe una specie di "sacralizzazione del crimine" in chiave paternalistico-eversiva, incentrata sulla violenza come strumento di riscatto, sull'indottrinamento e sul culto esasperato della sua figura. "Raffaele Cutolo - spiegò un abitante di Ottaviano al giornalista Giuseppe Marrazzo - aiuta questa gente che è stata bistrattata sia dai politici sia dalla magistratura, si mette sempre a disposizione [...] ha istituito un ordine, lo facessero uscire fuori e vedessero l'ordine che Raffaele Cutolo è capace di istituire in questa terra che muore di fame". "È il nostro santo protettore", rincarò il suo vicino. "Un delinquente nato" fu la più realistica definizione di Galasso, "uno che fa il delinquente dal mattino alla sera, che pratica quei modi antisociali, asociali, quei modi di approfittare del miserabile, dell'ignorante per scopi suoi". Molto giocò in questa mitologia la fama di Cutolo, da lui stesso perseguita concedendo interviste e trasformando le aule di tribunale in palcoscenici, fama ancor più amplificata dal libro di Marrazzo Il camorrista. Vita segreta di Raffaele Cutolo (Pironti editore, 1984) e dalla celeberrima pellicola di Tornatore ad esso ispirata.

La Nco fece registrare come nessun'altro sodalizio l'interscambio di modelli, simboli e slogan avutosi al tempo tra mafie e violenza politica: "i criminali inclini a pensare in grande - ha scritto Salvatore Lupo - impararono dalle gesta dello stragismo nero e del terrorismo rosso, riportate con grande evidenza dai media, o direttamente nelle comuni frequentazioni carcerarie, che il potere ufficiale poteva essere condizionato anche dall'intimidazione, oltre che dai consueti scambi di favore". La sigla Nco, ad esempio, richiamava le sigle politiche dell'epoca; estremistico, però, era soprattutto l'approccio dei sostenitori: "la camorra - spiegò un ottavianese al cronista Luca Rossi - è prendersi quello che non hai mai avuto, il pane, il lavoro, la 
casa"; "ci prendiamo quello che non ci danno", rilanciò una ragazza, "ce lo prendiamo con la forza". Quanto al programma dell'organizzazione, esso prevedeva, sostanzialmente, la reductio ad unum del crimine campano, ivi comprendendo le sue sezioni precedentemente confluite in Cosa nostra, facenti capo ai Nuvoletta di Marano e agli Zaza di Napoli (ancora Sales ha parlato in proposito di "mafizzazione" della camorra). Non stupisce che il progetto suscitasse vaste ostilità, e particolarmente quella di Carmine Alfieri e Pasquale Galasso, ai quali Cutolo fece uccidere un fratello ciascuno. Gli anticutoliani si federarono dunque nella cosiddetta Nuova famiglia (Nf), sfidando e vincendo il loro avversario al prezzo di centinaia di cadaveri. Proprio la violenza, introdotta su larga scala dal terrorismo politico, crebbe spaventosamente in quegli anni a opera della criminalità organizzata: si stima che i morti per mafia ammontino nel periodo 1975-1992 alla cifra mostruosa di 5000-6000. Nel solo 1991 si ebbero 700 delitti mafiosi, cifra di molto superiore a quella dei morti per cause politiche del periodo 1969-85.

Questa violenza va però letta sul versante camorristico alla luce, oltre che dello schema terroristico, del combinato disposto del business degli appalti prodotto dal terremoto campano del 1980 e della clamorosa vicenda Cirillo. Alle amministrazioni locali fu concesso di gestire in autonomia i fondi d'emergenza, circa 50.000 miliardi di lire, senza regole e senza controlli. I gruppi camorristici profittarono dell'affare, spartendosi o contendendosi il mercato degli appalti, comunque accrescendo il proprio potere. Quanto a Cirillo, esponente Dc napoletano, venne rapito nell'aprile del 1981 dalle Brigate rosse e - contrariamente ad Aldo Moro, per cui valse la linea della fermezza - liberato mercé una trattativa tra Stato e rapitori coinvolgente leader democristiani, agenti degli apparati di sicurezza e, nel ruolo di grande mediatore, lo stesso Cutolo. A tutto questo si richiamò Galasso per spiegare la tendenza cutoliana a colpire esponenti delle istituzioni: "da questo accordo tra Cutolo, quei politici, i servizi segreti e tutti gli apparati dello Stato che hanno concordato la liberazione di Cirillo, Cutolo prende il potere assoluto [...] quindi ci aggredisce ammazzando Salvatore Alfieri, perché pensava che lui potesse tutto. Nella stessa epoca della morte di Salvatore Alfieri, Cutolo aggredisce mio fratello, i vari elementi che rappresentavano degnamente lo Stato, tra i quali Lamberti, Gagliardi, Ammaturo, Salvi e carabinieri e poliziotti". 
Galasso spiegò come l'escalation seguisse una logica dimostrativa, cioè funzionale alla competizione inter-camorristica: "ricordo l'omicidio del commissario Ammaturo [che alcune fonti dicono ucciso dalle Br su mandato di Cutolo] mi sembra vice questore, di cui nel nostro gruppo, all'epoca, parlavamo un po', perché, per quanto mi risulta, l'Ammaturo non aveva fatto nulla di grave per meritarsi quella morte, che era per noi inspiegabile. Quindi, quello era un messaggio importante che Cutolo mandava a noi altri, per mostrare che non aveva limiti e frontiere. Come questo, posso dire di tanti altri casi”. Significativo, poi, che il super-pentito vedesse nell'offensiva cutoliana la mentalità mafiosa, "secondo la quale se uno dà fastidio bisogna eliminarlo". Stando alla sua versione, infatti, quell'arroganza e quella ferocia che a Palermo seminavano vittime tra funzionari di pubblica sicurezza, magistrati e politici sarebbero giunte anche a Napoli se Cutolo non fosse stato fermato: "voglio dirle che se andava avanti questa mentalità di mafia di Cutolo e di Nuvoletta, Napoli sarebbe diventata uguale a Palermo. Non faccio riferimento a chiacchiere ma agli attentati che ci sono stati: Salvi, il direttore del carcere di Poggioreale, è morto solo perché probabilmente ha avuto un alterco con Cutolo".

Insomma, le vicende di cui Galasso dà conto, qui schematicamente richiamate, restano emblematiche delle dimensioni, del peso e del potere conseguiti dal crimine organizzato nell'Italia degli anni ottanta: resta da capire come il sistema politico abbia potuto permettere tali degenerazioni. Le pagine che seguono forniscono un contributo in questa direzione, dando della nostra storia recente un'immagine meno “monumentale”, ma un po' più realistica. 


\section{Nota bibliografica}

Sul protagonismo delle mafie negli anni Ottanta cfr. John Dickie, Mafia Republic. Italy's Criminal Curse: Cosa nostra, Camorra and 'Ndrangheta from 1946 to the Present, Sceptre (trad. it: Mafia republic. Cosa Nostra, camorra e 'ndrangheta dal 1946 ad oggi, Laterza, Roma-Bari, 2013); Salvatore Lupo, Le mafie, in Storia dell'Italia repubblicana, III, L'Italia nella crisi mondiale. L'ultimo ventennio, 2, Istituzioni, politiche, culture, Einaudi, Torino, 1997, pp. 241 sgg.; Enzo Ciconte, Storia criminale. La resistibile ascesa di mafia, 'ndrangheta e camorra dall'Ottocento ai giorni nostri, Rubbettino, Soveria Mannelli, 2008. Quanto alla camorra in età repubblicana si vedano Francesco Barbagallo, Storia della camorra, Laterza, Roma-Bari, 2008; Luciano Brancaccio, I clan di camorra. Genesi e storia, Donzelli, Roma, 2017; Luciano Brancaccio, Carolina Castellano (a cura di), Affari di camorra. Famiglie, imprenditori e gruppi criminali, Donzelli, Roma, 2015; Gabriella Gribaudi (a cura di), Traffici criminali. Camorra, mafia e reti internazionali dell'illegalità, Bollati Boringhieri, Torino, 2009. La citazione di Isaia Sales è tratta dal suo La camorra, le camorre, Editori Riuniti, Roma, 1988, p. 155; le interviste a Ottaviano di Luca Rossi dal suo Camorra: un mese a Ottaviano, il paese in cui la vita di un uomo non vale nulla, Mondadori, Milano, 1982, pp. 76 82; quelle di Joe Marrazzo sono consultabili online su Raiplay. Per la citazione di Salvatore Lupo si veda Id., Antipartiti, Il mito della nuova politica nella storia della Repubblica (prima, seconda e terza), Donzelli, Roma, 2013, pp. 170-71, da cui ho tratto le stime sui delitti mafiosi (p. 170). Il concetto di "sacralizzazione del crimine" trae spunto da quello di "sacralizzazione della politica" formulato da Emilio Gentile per il fascismo italiano: si veda in proposito il suo Il culto del littorio. La sacralizzazione della politica nell'Italia fascista, Laterza, RomaBari, 1998. Sulla figura di Cutolo come personaggio "cross-mediale" rimando a Marcello Ravveduto, Lo spettacolo della mafia. Storia di un immaginario tra realtà e finzione, Edizioni Gruppo Abele, Torino, 2019; su quella di Pasquale Galasso a Gigi Di Fiore, Io, Pasquale Galasso: da studente in medicina a capocamorra, Pironti, Napoli, 1994. Sul caso Cirillo, infine, cfr. Isaia Sales, Ciro Cirillo, in Aa. Vv., Cirillo, Ligato e Lima: tre storie di mafia e politica (a cura di Nicola Tranfaglia), Laterza, RomaBari, 1994 e la sentenza del giudice Alemi in Vincenzo Vasile (a cura di), L'affare Cirillo, Editori Riuniti, Roma, 1989. 
Audizione del collaboratore di giustizia Pasquale Galasso, in Commissione parlamentare sul fenomeno della mafia e sulle associazioni criminali similari, 51, seduta di martedì 13 luglio 1993, pp. 2223-2260.

PRESIDENTE. Signor Galasso, la Commissione le rivolgerà mio tramite una serie di domande che cominciano da quelle di carattere biografico e proseguono sui temi della camorra ${ }^{1}$. La invito a dire alla Commissione il suo nome e la sua data di nascita.

PASQUALE GALASSO. Mi chiamo Galasso Pasquale e sono nato il 17 maggio 1955.

PRESIDENTE. Quali scuole ha frequentato?

PASQUALE GALASSO. Ho fatto fino al secondo anno di medicina a Napoli.

PRESIDENTE. Quale diploma aveva?

PASQUALE GALASSO. Ho studiato presso le scuole dei fratelli cristiani di San Giovanni Battista de La Salle in Benevento; ho preso la maturità tecnica e poi mi sono iscritto alla facoltà di medicina dell'università di Napoli.

PRESIDENTE. E ha frequentato per due anni?

PASQUALE GALASSO. Sì.

PRESIDENTE. Quale attività lavorativa ha svolto fino a quello che possiamo definire il suo arresto finale?

\footnotetext{
${ }^{1}$ I nominativi sostituiti con la parola OMISSIS sono coperti da segreto istruttorio.
} 
PASQUALE GALASSO. Mi sono occupato dell'impresa familiare.

PRESIDENTE. Che impresa era?

PASQUALE GALASSO. Diciamo industriale, di trasformazione degli autocarri FIAT, impresa creata da mio padre nel dopoguerra e fino al momento della mia collaborazione; oggi è chiusa.

PRESIDENTE. Si è occupato anche di altri tipi di attività legali, oltre che dell'impresa di suo padre?

PASQUALE GALASSO. Sì.

PRESIDENTE. Cioè?

PASQUALE GALASSO. Talvolta, contestualmente all'attività principale di mio padre, abbiamo fatto investimenti immobiliari (costruzioni, società di commercializzazione).

PRESIDENTE. Quando lei è diventato camorrista?

PASQUALE GALASSO. Dovrei premettere un fatto. Nel 1975, mentre frequentavo l'università, fui oggetto di un tentativo di sequestro nel corso del quale ammazzai due dei miei sequestratori.

PRESIDENTE. Lei girava armato?

PASQUALE GALASSO. No, disarmai uno di loro, sparai e ne ammazzai due; dopo una settimana mi sono costituito nelle mani dei giudici, sono stato portato al carcere di Poggioreale dove ho fatto giusto un anno di detenzione e dal quale sono uscito per eccesso di legittima difesa. Nel corso di questa detenzione ho conosciuto tutto il 
gotha criminale campano di quell'epoca, dal signor Cutolo ai maggiori referenti mafiosi che in Campania in quel momento gestivano tutti i traffici illeciti dei mafiosi. Questi signori sono: Michele Zaza, il fratello Salvatore, Nuvoletta, Sciorio, i Maisto.

PRESIDENTE. Allora erano tutti in carcere a Poggioreale?

PASQUALE GALASSO. Diciamo quasi tutti.

PRESIDENTE. Quindi?

PASQUALE GALASSO. Questi erano i maggiori esponenti criminali.

PRESIDENTE. Lei dunque ha conosciuto questi esponenti criminali durante il suo anno di detenzione a Poggioreale?

PASQUALE GALASSO. Sì, per il mio episodio mi trovai in un contesto di giungla dove c'era una sola legge, quella della prepotenza e della violenza. In quel momento ho cominciato ad avere in me la metamorfosi, anche se, più che altro, lo facevo per difendermi perché comunque avevo ammazzato due di questi.

PRESIDENTE. I suoi sequestratori appartenevano a qualcuna di queste bande camorristiche?

PASQUALE GALASSO. Sì, alle bande dell' hinterland napoletano.

PRESIDENTE. A quale gruppo?

PASQUALE GALASSO. Per la maggior parte del mio paese, un paio di gruppi camorristici dell'epoca.

PRESIDENTE. Vive a Poggiomarino? 
PASQUALE GALASSO. Sì.

PRESIDENTE. Quindi, se non ho capito male, lei dice: c'è stato un tentativo di sequestro; ha disarmato ed ha ucciso due di coloro che volevano sequestrarla; si è costituito dopo una settimana; ha fatto un anno di carcere a Poggioreale, dove sono detenuti i capi, o comunque i loro referenti...

PASQUALE GALASSO. Ho conosciuto tutte queste persone (diciamo i capi dell'allora gotha criminale campano), come tanti altri malavitosi. I maggiori esponenti di questi clan, che all'epoca facevano capo alle famiglie mafiose, erano Zaza, Nuvoletta, Sciorio, Maisto e tanti altri. Erano queste le maggiori famiglie referenti campane dei mafiosi. Ma poi c'era tutto il gotha criminale campano.

PRESIDENTE. Qual era la situazione di Poggioreale, allora? Cioè, un detenuto come lei, che arrivava, di fronte a quale situazione si trovava?

PASQUALE GALASSO. O subire, o eventualmente reagire ed accollarsi tutte le responsabilità.

PRESIDENTE. Subire che cosa?

PASQUALE GALASSO. Subire tutto, tutti gli abusi che si possono subire in un contesto d'ignoranza e di violenza.

PRESIDENTE. Lei con chi era in cella?

PASQUALE GALASSO. A Poggioreale, i primi due mesi sono stato in cella d'isolamento. Poi, fui trasferito al padiglione Milano, dove c'erano tutti quelli condannati per fatti di sangue. Poi, fui trasferito al centro clinico di Poggioreale. In un primo momento, fui messo in cella con elementi mafiosi: mi ricordo, all'epoca, Stefano Giaconia, appartenente ad una delle famiglie mafiose. Subito dopo, fui messo in una cella a fianco di quella di Cutolo Raffaele. 
PRESIDENTE. Il padiglione Milano era occupato da un gruppo camorristico in particolare o c'erano...

PASQUALE GALASSO. No, Poggioreale è diviso in tanti padiglioni. Nel padiglione Milano diciamo che c'erano tutti quelli che si erano macchiati di delitti di sangue.

PRESIDENTE. Indipendentemente dal gruppo cui appartenevano?

PASQUALE GALASSO. Indipendentemente dal gruppo cui appartenevano ed anche indipendentemente dallo strato sociale di provenienza. Badate bene, io ero studente universitario, avevo ammazzato due delinquenti dell'epoca, quindi fui messo in quello stesso padiglione dov'erano omicidi più diversi. In quel contesto, ho incominciato a reagire. Per non subire, è venuta fuori quella mia pessima personalità criminale.

PRESIDENTE. Quindi, lei diceva che praticamente in carcere ha conosciuto questi capi di varie famiglie camorristiche e che è stato vicino a Cutolo. Ma torniamo alla domanda di prima: come è diventato camorrista?

PASQUALE GALASSO. In questo contesto, dopo un anno che sono uscito per eccesso di legittima difesa, sono tornato a Poggiomarino, presso i miei familiari. Subito dopo, sono venuto a conoscenza che mio padre da tanti anni subiva estorsioni dai vari gruppi camorristici della zona. Il mio povero papà cercava di barcamenarsi tra l'accettare una volta ed il rifiutare un'altra volta queste piccole estorsioni. Eravamo una famiglia numerosa. Papà teneva più a difendere noi figli piccoli, e con enormi sforzi ha cercato sempre di darci un'altra vita. Purtroppo, debbo dire, perché oggi me ne sono accorto, che mio padre desiderava fare di me un laureato. Purtroppo, sono diventato un criminale. Una volta uscito, tornai a Poggiomarino. Diciamo che questi delinquenti della zona continuavano a dare fastidio ai miei familiari e a me particolarmente (hanno cercato di fare di tutto per ammazzarmi). Mio fratello Nino, buonanima, era una persona perbene, un imprenditore serio, amato da tutti. Hanno 
sempre cercato di invitarmi ad andarmene da Poggiomarino, da Napoli. Hanno sempre cercato di allontanarmi da loro, ma era più forte di me, perché papà e la mia famiglia per me erano tutto. In quel contesto, ho cercato di stare attento. Nel giro di un paio d'anni, ho subito diversi attentati, e man mano, per difendermi, per non lasciare il mio paese e la mia zona, per non allontanarmi ho stretto rapporti inizialmente amicali con qualche grosso personaggio malavitoso della zona cui facevo riferimento. Cercavo di spiegarmi dicendo: non è possibile che io debba subire ancora da questa gentaglia. In particolare, ho stretto rapporti con Salvatore Alfieri, fratello di Carmine Alfieri, una persona squisita, dico squisita e perbene malavitosamente.

PRESIDENTE. Carmine Alfieri era insediato a Poggiomarino?

PASQUALE GALASSO. Anche Carmine Alfieri l'ho conosciuto in carcere, nel 1976, quando ero detenuto per il duplice omicidio. Ma la persona che ho più frequentato, una volta uscito dal carcere, è Salvatore Alfieri, che all'epoca era uno dei capicamorra della zona.

PRESIDENTE. Quindi, lei non diventa camorrista in carcere?

PASQUALE GALASSO. No.

PRESIDENTE. Fuori del carcere?

PASQUALE GALASSO. Durante il mio trascorso a Poggioreale, Cutolo, Michele Zaza ed un po' tutti mi corteggiavano. Più di una volta mi hanno invitato a legarmi a loro, ma io ho sempre riferito, a Cutolo anche in prima persona, che l'unica cosa cui tenevo era mio padre e la mia famiglia. Non cercavo altre famiglie. Quindi, in quell'anno di detenzione con Cutolo, rapporti normali. Ma se di tanto in tanto litigavo con altri detenuti, era Cutolo ad intervenire in mio favore.

PRESIDENTE. Ci fu una cerimonia particolare di affiliazione alla camorra? 
PASQUALE GALASSO. No. Volevo spiegarvi, appunto, come mi sono poi trovato in questo ambiente criminale. Se volete, posso anche essere breve...

PRESIDENTE. No, dica.

PASQUALE GALASSO. Una volta uscito, una volta che continuavano queste questioni ai danni miei e dei miei familiari, ho stretto amicizia con Salvatore Alfieri, cercando di farmi capire da lui, in qualità della sua personalità camorristica, dicendogli anche che non potevo andarmene da Poggiomarino, che io ed i miei familiari non ce ne andavamo e che, eventualmente, avrei preferito essere ammazzato. In quegli anni in cui Salvatore Alfieri mi ha dato una mano a chiarirmi con questi altri elementi malavitosi, ho subìto alcuni attentati. Ma nel 1978 attentarono alla vita di mio padre e lo ferirono alla testa.

PRESIDENTE. In che periodo del 1978?

PASQUALE GALASSO. Dovrebbe essere il 1978-1979, se ricordo bene.

PRESIDENTE. Fra la fine del 1978 ed i primi del 1979?

PASQUALE GALASSO. 1978-1979.

PRESIDENTE. Spararono a suo padre?

PASQUALE GALASSO. Sì, sotto casa attentarono alla vita di mio padre. In questo contesto, ho frequentato Salvatore Alfieri, e tramite lui all'epoca ho conosciuto quasi tutti (in parte li avevo già conosciuti in carcere). Quindi, ho cercato di difendermi. In questo rapporto di amicizia con Salvatore Alfieri, nel 1978-1979 esce dal carcere Carmine Alfieri. Ci rivediamo, ne parliamo, stringiamo amicizia. Nel 1980-1981 gli Alfieri erano legati a Lorenzo Nuvoletta, ma non da rapporti di affiliazione ma amicali, rapporti stretti. Il Nuvoletta avrebbe preferito che io e gli Alfieri ci 
legalizzassimo mafiosi, appartenenti alla sua... ai Nuvoletta, i Nuvoletta-corleonesi. Noi prendevamo tempo perché non vedevamo chiaro i Nuvoletta nella posizione contro Cutolo. Siamo negli anni 1980-1981. Gli Alfieri erano aggiornati che i Nuvoletta hanno sempre fatto una politica criminale doppiogiochista, cioè per sopravvivere a tutti gli eventi, alle epoche criminali, semmai si erano macchiati di tradimento nei confronti dei loro più stretti uomini di fiducia. Quindi, queste erano macchie che a noi principalmente agli Alfieri, a Carmine Alfieri - risultava e quindi al loro invito di legalizzarci mafiosi noi abbiamo preso sempre tempo. Nel 1981 nasce la vicenda Cirillo, dove per me c'è il...

PRESIDENTE. Aspetti un attimo, prima di Cirillo una cosa che ci interessava capire è se c'è una cerimonia di sua affiliazione alla camorra o avviene così?

PASQUALE GALASSO. No, nel mio gruppo non c'è stata cerimonia. Il gruppo di Carmine Alfieri nasce con Pasquale Galasso, Enzo Moccia, principalmente. Enzo Moccia è un'altra persona che ho conosciuto nel carcere.

PRESIDENTE. Poi il nome forse non è Enzo.

PASQUALE GALASSO. E' Angelo, Angelo Moccia. E' un giovane come me; diciamo che ha avuto la sventura... quasi la mia stessa storia. Gli ammazzano il padre mentre sta nel carcere (io l'ho conosciuto nel carcere di Poggioreale). Una persona che credo, se sceglierà la mia stessa strada, un domani potrebbe ricrearsi una vita per bene, nonostante si è macchiato, come me, di tanti delitti orrendi.

PRESIDENTE. Lei stava dicendo che nel 1981 ci fu...

PASQUALE GALASSO. No, per portarvi da quando è nata questa organizzazione con Carmine Alfieri. Diciamo che è incominciata a nascere con l'avvento di Cutolo. Nel 1980-1981 gli Alfieri principalmente erano preoccupati di questa prepotenza di Cutolo. Cercavamo di difenderci, avevamo quei rapporti con Lorenzo Nuvoletta, che ancora una volta speculavano su queste tensioni che creava Cutolo. Alla fine, dopo 
riunioni diverse riunioni presso i Nuvoletta, riunioni di tregua, di pace, che Cutolo doveva lasciare tranquilli un po' tutti gli ambienti, tutti i gruppi camorristici campani... i Nuvoletta, che facevano da intermediari, da mediatori, però dopo si è scoperto che loro facevano ancora una volta il doppio gioco. A fine 1981 muore Salvatore Alfieri ammazzato dai cutoliani. Il 15 gennaio $1982 \mathrm{mi}$ ammazzano, per vendetta trasversale, mio fratello Nino; come le dicevo, una persona per bene, invalido. Io da quel momento giurai su mio fratello Nino che l'avrei vendicato e da quel momento ho incominciato a stringere un rapporto di fedeltà tra me e gli Alfieri, Enzo Moccia, inizialmente, e poi altri nostri aggregati, senza affiliazione, questo volevo portare, senza cerimonia: è un rapporto amicale stretto sul sentimento dell'amicizia.

PRESIDENTE. Questo a differenza di quel che succedeva con Cutolo, perché Cutolo faceva le cerimonie.

PASQUALE GALASSO. Cutolo e un po' tutti i gruppi camorristici, quasi tutti, fanno iniziazione, non so come, noi li abbiamo sempre derisi, io e vi parlo di Enzo Moccia.

PRESIDENTE. Lei è stato mai affiliato a Cosa nostra?

PASQUALE GALASSO. No, seppure ho avuto diverse proposte, come le dicevo, dai vari esponenti mafiosi campani.

PRESIDENTE. Quando era in carcere o anche dopo?

PASQUALE GALASSO. Quando ero nel carcere e pure fuori. Mi ricordo che Zaza da Napoli, quando avevo problemi a Poggiomarino, nella mia zona, lui era aggiornato tramite il suo referente... Mario Fabbrocino e Pasquale Russo, i suoi referenti nell' hinterland, sono venuti più di una volta a Poggiomarino ad offrirmi la loro solidarietà, però, mi invitavano, mi dicevano che mi dovevo legalizzare ma io come vi ho detto... 
PRESIDENTE. Legalizzare che vuol dire?

PASQUALE GALASSO. Vuol dire associarsi al loro gruppo che è un gruppo mafioso. Zaza fa parte della famiglia dei Greco di Ciaculli.

PRESIDENTE. Ho capito. Lei che tipo di automobili usava quando era in libertà?

PASQUALE GALASSO. Diciamo che noi come attività facevamo la trasformazione di autocarri e poi la vendita di automobili, quindi avevamo una concessionaria, un autosalone.

PRESIDENTE. No, voglio dire questo, dagli interrogatori che abbiamo visto...

PASQUALE GALASSO. Papà non mi ha fatto mai mancare niente. Da quando avevo 20 anni ho usato Ferrari, Porsche e macchine di piccola cilindrata.

PRESIDENTE. Cosa le dava questa ricchezza? Soltanto il commercio di autoveicoli oppure anche altro?

PASQUALE GALASSO. Non ho capito.

PRESIDENTE. Cosa le dava questa ricchezza? Soltanto il commercio di autoveicoli o anche altre attività?

PASQUALE GALASSO. Vorrei capire bene, signor presidente.

PRESIDENTE. Credo che non tutti possiedano Ferrari, Porsche, eccetera. Possedere questo tipo di macchine è in genere segno di una particolare ricchezza.

PASQUALE GALASSO. Sì. 
PRESIDENTE. Ecco, le ragioni di questa ricchezza erano soltanto il lavoro che facevate sui veicoli industriali e sulle macchine oppure anche altro?

PASQUALE GALASSO. Solo quello. Dal dopoguerra mio padre... Inizialmente era commerciante di cereali, negli anni subito dopo la guerra. Poi, con il proprio lavoro e con le speculazioni, con investimenti immobiliari, si è creato una ricchezza che poi ci ha fatto vivere agiatamente.

PRESIDENTE. Lei aveva una villa bunker?

PASQUALE GALASSO. No, noi tenevamo la nostra attuale abitazione in Poggiomarino, che poi è nel comune di Scafati, dove c'è un muro recintato. Inizialmente, papà l'ha sempre avuto per la custodia degli autocarri. Mi ricordo che 20, 30 anni fa mio padre ha subìto diversi furti di autocarri. Quindi, era un muro. Certamente, dopo l'omicidio di mio fratello Nino, devo dire il vero, su questo muro abbiamo messo un'inferriata alta circa un metro. Questo è tutto.

PRESIDENTE. Ci sono telecamere, cose di questo genere o no?

PASQUALE GALASSO. Ha la telecamera all'ingresso ma come un po' tutte le aziende. Certamente, le dico, dopo l'omicidio di mio fratello Nino, il cancello... se veniva un cliente, il portiere, se lo conosceva, lo faceva entrare o eventualmente gli chiedeva documenti.

PRESIDENTE. Lei si è mai occupato di lotto clandestino?

PASQUALE GALASSO. No, mai.

PRESIDENTE. Lei si è mai occupato di traffico di stupefacenti?

PASQUALE GALASSO. No, non ci siamo mai occupati di traffico di stupefacenti e credo neanche Alfieri, almeno come lui sempre mi ha detto. 
PRESIDENTE. Quando dice "non ci siamo" che vuol dire?

PASQUALE GALASSO. Io e il mio gruppo.

PRESIDENTE. Come mai, se tutti se ne occupavano, voi proprio no?

PASQUALE GALASSO. Diciamo che principalmente dopo l'omicidio Casillo non era importante trafficare in stupefacenti, c'era ben altro da occuparsi.

PRESIDENTE. Cosa è il ben altro?

PASQUALE GALASSO. Gli appalti.

PRESIDENTE. E rendevano quanto gli stupefacenti?

PASQUALE GALASSO. Credo pure maggiormente.

PAOLO CABRAS. Non ha mai fatto contrabbando di sigarette?

PASQUALE GALASSO. Credo che Alfieri in prima persona, almeno quello che lui...

PAOLO CABRAS. Qual era la ragione sociale del gruppo Alfieri?

PASQUALE GALASSO. Diciamo, gli appalti e poi tutti gli altri traffici, tutti gli altri interessi...

PAOLO CABRAS. Gli appalti, dopo l'uccisione di Casillo, io dico prima.

PASQUALE GALASSO. Prima non lo so. Non so cosa abbia fatto, perché poi certamente non ha la mia stessa età. 
PRESIDENTE. Comunque, le domande riguardavano lei. Lei dice: "non ho mai fatto lotto clandestino, non ho mai fatto traffico di stupefacenti". Non ha mai fatto traffico di stupefacenti perché le altre attività rendevano già sufficientemente. E' questo il tipo di ragionamento?

PASQUALE GALASSO. Sì.

PRESIDENTE. L'omicidio di suo fratello Nino fu il 15 o il 21 gennaio 1982?

PASQUALE GALASSO. 21 gennaio 1982.

PRESIDENTE. Perché lei ha detto il 15 prima.

PASQUALE GALASSO. Ho sbagliato, mi scusi.

PRESIDENTE. Fu una ritorsione dei cutoliani?

PASQUALE GALASSO. Sì.

PRESIDENTE. A che cosa?

PASQUALE GALASSO. Diciamo, all'omicidio di Salvatore Alfieri, il 26 dicembre 1981, segue l'omicidio di Alfonso Catapano, in Poggiomarino, responsabile dell'omicidio Alfieri. Dopo l'omicidio Catapano, dei primi giorni - credo il 6 - del gennaio 1982, segue l'omicidio di mio fratello Nino.

PRESIDENTE. Praticamente, c'è uno scontro tra il gruppo Alfieri ed il gruppo di Cutolo, è così?

PASQUALE GALASSO. Sì. 
PRESIDENTE. Il gruppo Cutolo uccide Salvatore Alfieri; voi uccidete Catapano e quelli uccidono suo fratello. E' questa la dinamica?

PASQUALE GALASSO. Sì.

PRESIDENTE. Perché c'era questo scontro tra voi e Cutolo? Aspetti, magari ci arriviamo tra un attimo. Lei si è mai occupato di un consorzio PRO.CAL. per il mercato del calcestruzzo?

PASQUALE GALASSO. No, ma lo conosco.

PRESIDENTE. Di cosa si tratta?

PASQUALE GALASSO. E' un consorzio di calcestruzzi, dove vi è l'intreccio, come al solito, tra politici, camorra e imprenditori.

PRESIDENTE. Può spiegarlo bene?

PASQUALE GALASSO. Sì; per quanto mi risulta, il consorzio PRO.CAL. è stato costituito con appoggi politici. Il consorzio sborsa la tangente prima ai politici e poi...

PRESIDENTE. Andiamo con ordine: in che epoca siamo all'incirca?

PASQUALE GALASSO. Se non erro, nel 1988-1989.

PRESIDENTE. Per quale ragione si costituisce questo consorzio?

PASQUALE GALASSO. Si costituisce perché c'è un marasma totale nel settore del calcestruzzo, per quanto riguarda i prezzi, le modalità e tante altre cose, ed anche perché i calcestruzzi erano sotto il tiro della Guardia di finanza, dei carabinieri e dei giudici inquirenti. Il consorzio, quindi, doveva mostrare quella facciata apparentemente legale, superiore a ogni intrigo, intreccio, interesse, collusione fra 
camorra e imprenditori; si pensò bene, quindi, di fare questo consorzio PRO.CAL. Così come doveva nascere anche un altro consorzio: inizialmente il PRO.CAL. doveva essere unico per Salerno e Napoli ma successivamente si doveva dividere. Oltre al consorzio di Napoli, doveva nascere un consorzio a Salerno.

PRESIDENTE. E nasce poi il consorzio a Salerno?

PASQUALE GALASSO. No, non nasce.

PRESIDENTE. Lei ha fatto riferimento ad una situazione di confusione nel mercato del calcestruzzo: l'onorevole Ricciuti chiede se l'importazione di cemento era clandestina o legale.

PASQUALE GALASSO. Era apparentemente legale, ma vi erano dietro interessi camorristici. Mi spiego meglio: per quanto mi risulta, vi erano dei greci collusi con la camorra e con qualche politico che portavano le forniture di cemento a Napoli.

PRESIDENTE. Erano forniture clandestine?

PASQUALE GALASSO. No, apparentemente erano legali; venivano sdoganate, credo, nei porti di Napoli e di Salerno.

PRESIDENTE. Era solo il disordine che vi indusse a creare questo consorzio, o vi furono occasioni particolari?

PASQUALE GALASSO. No, principalmente il disordine ed anche perché si vedeva che le forze dell'ordine stavano addosso ai vari piccoli calcestruzzai.

PRESIDENTE. Che erano già legati alla camorra?

PASQUALE GALASSO. Sì. 
PRESIDENTE. Lei ha parlato di protezioni politiche dietro al consorzio: quali sono?

PASQUALE GALASSO. Io di questo consorzio non mi sono occupato, ma per quanto mi risulta sono quasi sempre gli stessi.

PRESIDENTE. Cioè?

PASQUALE GALASSO. Maggiormente la corrente dorotea, facente capo a Gava.

PRESIDENTE. Cosa aveva fatto in relazione a questo consorzio?

PASQUALE GALASSO. Dietro al consorzio era principalmente l'onorevole Boffa, uno dei principali politici che dava protezione ed assistenza politica.

PRESIDENTE. Spieghi bene cosa faceva Boffa.

PASQUALE GALASSO. Le ripeto che non ho vissuto in prima persona le vicende del consorzio PRO.CAL. ma, per quanto mi risulta dalla mia organizzazione, da Carmine Alfieri, da Mimmo e Luigi Romano, imprenditori nostri associati, era per volontà di questi politici che nasceva il consorzio PRO.CAL.

PRESIDENTE. Che utilità avevano questi politici?

PASQUALE GALASSO. Avevano i loro interessi, sia di voto, sia di arricchimento personale e talvolta di partito, come eventualmente potrebbero far passare questi finanziamenti. Una volta superata questa prima fase con i politici, il consorzio PRO.CAL. contattava quasi tutti i gruppi camorristici, concedendo 2 mila lire al metro cubo sulle forniture. Nacque una guerra, principalmente fra il consorzio ed il gruppo camorristico napoletano di Contini, Licciardi, Mallardo, in quanto pretendevano 5 mila lire al metro cubo, invece di 2 mila lire. Questo fatto fece pure incrinare un po' i rapporti fra il nostro gruppo, capeggiato da Carmine Alfieri, e lo stesso clan Contini, Licciardi, Mallardo... 
PRESIDENTE. Che erano quelli che stavano a Napoli.

PASQUALE GALASSO. Sì, tant'è vero che inizialmente tutta la cifra della tangente del consorzio PRO.CAL. veniva portata nelle mani di un imprenditore nostro associato...

PRESIDENTE. Chi era?

PASQUALE GALASSO. Luigi Romano; poi Alfieri pensava a dividerla fra i gruppi camorristici a noi associati. Da quel momento, si sciolse questa intesa: quindi il gruppo Licciardi, Contini, Mallardo proseguì per la sua strada nei riguardi del consorzio PRO.CAL. ed invece al gruppo Alfieri stavano bene le solite 2 mila lire al metro cubo.

PRESIDENTE. Lei ha detto: superata la prima fase, come se dapprima si fossero acquietati i politici e poi si fosse passati a pagare la camorra.

PASQUALE GALASSO. Sì.

PRESIDENTE. Come si acquietavano i politici?

PASQUALE GALASSO. Diciamo con l'intesa: una volta soddisfatti i propri interessi....

PRESIDENTE. Come si soddisfacevano questi interessi?

PASQUALE GALASSO. Io credo con una tangente prefissata, ma non vi so dire i particolari.

PRESIDENTE. Quali vantaggi vi offrivano i politici in cambio del pagamento da parte vostra di una tangente?

PASQUALE GALASSO. Essenzialmente un vantaggio in termini di voti. 
PRESIDENTE. Questo era per i politici, ma a voi cosa davano?

PASQUALE GALASSO. Vi era, diciamo, l'intreccio, la commistione, la simbiosi, l'accordo di tranquillità tra noi e i politici.

PRESIDENTE. Nel PRO.CAL., per quanto lei sa - visto che dice di non essersi direttamente interessato - i politici che funzione avevano? Che ruolo svolgevano? Perché voi dovevate dare loro una tangente e fare avere i voti? In cambio cosa vi davano?

PASQUALE GALASSO. La protezione, almeno quella politica, la solidarietà per ogni problema, per quanto riguarda sia noi gruppo camorristico, sia gli imprenditori facenti capo al PRO.CAL.

PRESIDENTE. Cosa vuol dire protezione politica agli imprenditori?

PASQUALE GALASSO. Loro sovrintendevano un po' a tutto: se domani mattina il PRO.CAL. o il singolo imprenditore ha un problema, si rivolge al politico che sta dietro al PRO.CAL. e che cerca di risolvere il problema.

PRESIDENTE. Quale può essere il problema?

PASQUALE GALASSO. Un favore qualsiasi: per esempio, non so, un certificato antimafia per un imprenditore facente capo al PRO.CAL., oppure ben altro favore. Quindi, un rapporto, un'intesa, una copertura politica.

PRESIDENTE. Quindi, se comprendo bene, non vi era un aspetto specifico?

PASQUALE GALASSO. Essenzialmente, se manca la protezione politica, il consorzio PRO.CAL. non nasce, come non è nato, almeno per quanto mi risulta, un analogo 
consorzio nel salernitano, dove è mancata la volontà specifica di qualche politico a livello nazionale.

MARIO CLEMENTE MASTELLA. Vorrei sapere da chi abbia ricevuto queste notizie su PRO.CAL.

PRESIDENTE. La invito a rispondere a questa domanda.

PASQUALE GALASSO. Dalla mia associazione sempre facente capo a Carmine Alfieri e di cui io ero un capo storico perché, insieme ad Alfieri, appartenevo al direttivo. Nel 1988 sono stato socio di Terracciano Francesco della Calcestruzzi vesuviana, quindi interessato a questa società insieme ad altri imprenditori calcestruzzai.

PRESIDENTE. La Calcestruzzi vesuviana faceva parte del consorzio?

PASQUALE GALASSO. Sì, ultimamente è entrata anch'essa mentre io sono uscito dalla società. Erano due calcestruzzi, quindi tutto quello che mi risulta è direttamente in virtù del mio interessamento in qualità di socio della Calcestruzzi vesuviana e in qualità di amico di altri grossi calcestruzzai della zona di Napoli.

MARIO CLEMENTE MASTELLA. Vorrei sapere se Boffa è un politico locale e perché il consorzio non sia nato nella provincia di Salerno. Forse perché è mancato il riferimento a politici nazionali?

PASQUALE GALASSO. Vorrei fare a meno di rispondere a questa domanda perché sono in corso indagini coperte dal segreto istruttorio. Preferirei non rispondere.

PRESIDENTE. Poiché lei ha parlato di un signor Boffa come di colui che teneva le fila, l'onorevole Mastella le chiedeva chi fosse dietro questo consorzio.

PASQUALE GALASSO. Boffa è uno dei politici che ha organizzato il PRO.CAL., ha dato la copertura, l'assistenza politica, almeno iniziale, per la nascita di questo consorzio. 
PRESIDENTE. Lei poi ha aggiunto che a Salerno quest'associazione non è nata perché nessun politico nazionale ha dato la copertura, se non ho capito male.

PASQUALE GALASSO. Non è che non abbia dato la copertura, non si è arrivati a certi accordi o a certe volontà politiche, particolarmente di un grosso politico a livello nazionale del salernitano.

PRESIDENTE. Chi era?

PASQUALE GALASSO. Preferirei non rispondere, se possibile, perché vi è il segreto istruttorio.

PRESIDENTE. Questa Commissione ha interesse a capire bene quali siano le caratteristiche delle organizzazioni camorristiche. Abbiamo studiato in modo abbastanza approfondito quelle di Cosa nostra, della mafia siciliana, organizzazioni che conosciamo meglio rispetto alla camorra; ora invece abbiamo bisogno di capire bene come siano organizzati i gruppi camorristici. C'è un comando unico per tutte le organizzazioni della camorra o ciascuna si muove per conto proprio? Ovvero dipende da momento a momento?

PASQUALE GALASSO. Dopo la scomparsa di Bardellino Antonio...

PRESIDENTE. Può dire quando?

PASQUALE GALASSO. Maggio-giugno 1988. Dopo la scomparsa di quest'ultimo c'era l'intenzione di creare una cupola camorristica di tutti i clan vincenti, quelli più feroci della Campania, fra questi il nostro gruppo, quello di Licciardi, Mallardo, Contini e il clan cosiddetto dei Casalesi, quello che prima faceva capo a Bardellino. A questa intesa però non si è arrivati, anche per le tensioni che creavano altri gruppi che sentivano di rimanere esclusi. Ogni gruppo camorristico campano, a differenza di altri gruppi delinquenziali, è autonomo. 
PRESIDENTE. Quindi l'unico tentativo è stato quello ma non si è riusciti.

PASQUALE GALASSO. Non è riuscito, se pure c'era una forte intesa tra noi, il gruppo Licciardi, Mallardo, Contini, che sono maggiormente presenti a Napoli città e nella provincia (Marano, Giuliano).

PRESIDENTE. Perché la scomparsa di Bardellino vi indusse a cercare questa strada?

PASQUALE GALASSO. Perché secondo me Bardellino era un punto di riferimento per tutti i clan camorristici campani, eventualmente pure per quelli che non lo sopportavano; riusciva a mantenere con il suo carisma un certo equilibrio campano. Morto Bardellino...

PRESIDENTE. Morto o scomparso?

PASQUALE GALASSO. A me risulta che sia scomparso, ma è morto. Morto Bardellino, vi sono state diverse altre guerre di clan con centinaia di morti. Si stava eccedendo e quindi si è ritenuto opportuno creare una coalizione dei gruppi più feroci, quelli che erano più presenti sul territorio affinché tutte queste piccole faide, piccole guerre finissero, per arrivare ad una tranquillità, a quello che fa comodo ai gruppi camorristici di un certo rilievo e ai loro intrecci e raccordi con altri ambienti politici istituzionali. La tranquillità è una cosa importante, signor presidente; se manca è tutto difficile, almeno per il gruppo camorristico, per il camorrista e anche per tutto l'intero ambiente di zona.

PRESIDENTE. Cosa vuol dire la tranquillità?

PASQUALE GALASSO. Quando c'è la serenità.

PRESIDENTE. Quando non ci sono guerre? 
PASQUALE GALASSO. Quando non c'è guerra, quando non c'è niente si può lavorare tranquillamente, certi rapporti si possono curare meglio e si possono fare tutti i vari traffici serenamente, le forze dell'ordine non premono, non c'è esigenza, non ci sono preoccupazioni diverse.

PRESIDENTE. Com'è composto un gruppo camorristico? C'è un vertice, un gruppo che consiglia il vertice? Come si crea il vertice? Com'è organizzato?

PASQUALE GALASSO. In ogni gruppo camorristico c'è un capo, attorno al quale ci sono le sue persone più di fiducia, quelle che si distinguono nei momenti di tempesta, di guerra, vuoi per la loro ferocia vuoi per il loro saper fare, per la loro managerialità, per il loro dialogo oltre che con il settore delinquenziale anche con i vari altri strati sociali. Queste figure emergono, attorniano il capo, si crea un direttivo con il capo. In questo direttivo si decide tutto: i rapporti con questa o quell'altra persona...

PRESIDENTE. Ci sono gruppi di fuoco, come esistono in analoghe organizzazioni, cioè persone che fanno soltanto azioni di violenza?

PASQUALE GALASSO. Sì. Nel nostro gruppo all'inizio eravamo in pochissimi; dico inizialmente perché mi riferisco al periodo della guerra contro Cutolo che io e pochi altri miei amici mi sono sposato (volgarmente parlando). A questo punto, signor presidente, devo confessare una cosa: dopo la morte di mio fratello Nino cercavo la morte per liberarmi di ogni cosa; volevo morire, ma prima di morire volevo ammazzare chi aveva ammazzato mio fratello. Come me c'era qualche altro amico mio.

PRESIDENTE. Lei quindi faceva parte di questo gruppo particolarmente violento?

PASQUALE GALASSO. Sì, fino agli ultimi anni; invece altri gruppi camorristici hanno gruppi di fuoco, talvolta si servono di persone estranee, tossicodipendenti; diciamo che si va nella volgarità più assoluta, a seconda degli elementi che servono. Per 
esempio, mi risulta che per compiere omicidi altri gruppi camorristici si siano serviti di poveretti, di tossicodipendenti, che sono poi stati a loro volta uccisi.

PRESIDENTE. Quali gruppi, per esempio?

PASQUALE GALASSO. Ma... Gionta, lo stesso Nuvoletta. Un po' tutti. Principalmente Licciardi...

PRESIDENTE. Voi no, voi facevate direttamente...

PASQUALE GALASSO. No, noi no. I fatti, se non venivano fuori con la mia collaborazione, può darsi che morissero eternamente.

PRESIDENTE. Quindi, il capo del gruppo camorristico non è scelto da un gruppo di persone. In Cosa nostra avviene che...

PASQUALE GALASSO. Nella mia organizzazione, il capo, Carmine Alfieri, viene fuori dalla nomea degli Alfieri, dalla loro grossa fama di malavitosi da sempre: la famiglia degli Alfieri è presente nella nostra zona dal dopoguerra. Quindi, io ed i nostri amici, abbiamo sempre riversato aspetto ed ossequio verso questa persona appartenente ad una nota famiglia camorristica. Diciamo pure che nel frattempo Alfieri ha speculato sul nostro coraggio e sulle nostre figure.

PRESIDENTE. C'è una divisione del territorio per competenza dei singoli gruppi camorristici?

PASQUALE GALASSO. Sì, tutto il territorio campano. E' un po' - se posso permettermi - come fanno i politici: ci sono le varie correnti (questa è una cosa chiara, non sto dicendo menzogne)... Signor presidente, io vorrei essere il più chiaro possibile, perché voglio chiedere solo una cosa, principalmente a voi ed allo Stato italiano: di darmi la possibilità di rinascere, di riabilitarmi e di dimostrare allo Stato la mia valenza sociale. 
PRESIDENTE. La Commissione è qui che l'ascolta.

PASQUALE GALASSO. Voglio parlare con la massima chiarezza, senza sotterfugi o ipocrisie. Negli ultimi tempi, la camorra campana aveva suddiviso tutto il territorio della regione, un po' come fanno i politici, i quali sono presenti in tutte le zone: in una determinata zona, contestualmente alla presenza politica vi è quella del capocamorra o del referente camorrista. La Campania è suddivisa fra tanti gruppi malavitosi.

PRESIDENTE. E quali sono i gruppi per territorio?

PASQUALE GALASSO. Nel casertano prevalentemente opera il clan dei casalesi, oggi con a capo Schiavone, Bisognetti e qualcun altro.

PRESIDENTE. Questo a Caserta. Poi?

PASQUALE GALASSO. Sì, a Caserta il cosiddetto clan dei casalesi. A Napoli città (parlo delle figure prevalentemente presenti), Licciardi, Mallardo e Contini, fino ad arrivare nel giulianese, con un'intesa, equivoca ed ambigua tra di loro, con il clan Nuvoletta (Lorenzo Nuvoletta) e, contestualmente, tramite Giuseppe Mallardo, con il clan dei casalesi. Poi, ad Afragola, Casoria, Caivano, Frattamaggiore e Frattaminore il clan Moccia, miei amici. A Nola e ad Acerra vi sono altri gruppi che fanno capo a Mario Fabbrocino, ma è inutile specificare i nomi, perché si tratta di piccole famiglie malavitose (comunque, principalmente c'è l'antica famiglia dei Nuzzo). Nel nolano e nel vesuviano ci siamo noi, arriviamo nel salernitano, fino a giungere in Calabria. Ad Avellino c'è un altro gruppo facente capo a noi, quello di Sepe Marzio. Contestualmente, vi sono altri due gruppi, che sono in guerra oggi e da diversi anni, quello dei Graziano e quello dei Gava. Ciò vuol dire, per esempio, che una ditta che va a lavorare ad Avellino, se non intreccia rapporti con questi clan non può lavorare, anche se ha la protezione politica. 
PRESIDENTE. Quest'ultima non basta?

PASQUALE GALASSO. Non basta.

PRESIDENTE. Lei ha detto che la vostra influenza si estende fino in Calabria. Può spiegarci che intende dire?

PASQUALE GALASSO. Tutto il salernitano è coperto dai nostri clan che, principalmente, fanno capo al clan Maiale ed al clan Pecoraro, i quali sono legati ad Alfieri.

PRESIDENTE. Ed ora che Alfieri è arrestato, chi è il capo di questo clan?

PASQUALE GALASSO. C'è un gruppo che oggi fa capo a Sepe Marzio.

PRESIDENTE. Quindi, è lui che ha preso il posto...

PASQUALE GALASSO. Sepe Marzio, Autorino, i fratelli Russo, D'Avino e qualche altra persona.

PRESIDENTE. Quando un capo come Alfieri è arrestato, continua a comandare e a dirigere o no?

PASQUALE GALASSO. Sì, continua a comandare.

PRESIDENTE. Quindi, Sepe sarebbe una specie di rappresentante...

PASQUALE GALASSO. Certamente, Sepe con il direttivo.

PRESIDENTE. Ma il direttivo è una cosa formalizzata oppure nasce di fatto? Non so se ho formulato chiaramente la domanda. 
PASQUALE GALASSO. No, nasce appositamente, sono scelte persone...

PRESIDENTE. Li sceglie il capo?

PASQUALE GALASSO. Sì, il capo. Sono persone che già nel campo di guerra, durante le tempeste hanno dimostrato di saperci fare, vuoi per la ferocia, vuoi per altri requisiti.

PRESIDENTE. Quindi, c'è il capo che chiama attorno a sé quelli più capaci. Ma questo gruppo di persone più vicino al capo è una struttura in qualche modo formale o è di fatto? E' chiara la domanda?

PASQUALE GALASSO. No, è di fatto. Mi spiego: se altri accoliti o associati lo sanno? Sì, lo sanno, sono a conoscenza, sanno la valenza criminale di tutto il direttivo.

PRESIDENTE. Quando si decide un omicidio, chi lo decide?

PASQUALE GALASSO. Il capo con il direttivo. Ci sono persone che sono già state designate a morire: si aspetta il momento propizio, nel senso che queste persone potrebbero essere in carcere, non presenti in zona o non ben esposte; quando i fatti sono propizi, il capo e il direttivo decide come fare; si organizzano e scelgono anche i killer.

PRESIDENTE. Come si è andato organizzando attorno ad Alfieri il gruppo dirigente?

PASQUALE GALASSO. Inizialmente eravamo Alfieri, io, Enzo Moccia...

PRESIDENTE. Veniva chiamato Enzo ma il nome di battesimo è Angelo? E' così?

PASQUALE GALASSO. E' di famiglia che viene chiamato così. C'era Giuseppe Olivieri, dell'agro nocerinosarnese, del salernitano; poi, dopo sono venuti Giuseppe Ruocco, Ferdinando Cesarana (Sepe Marzio già esisteva prima, insieme a me e a Enzo Moccia, 
ma si trovava in carcere) e Giuseppe Autorino. Questo era il nostro gruppo direttivo. Poi, dopo, negli anni 1985-1986, si aggiungono a noi altri elementi mafiosi provenienti dal gruppo Zaza.

PRESIDENTE. Quanti erano, sostanzialmente, tutti quelli che giravano attorno ad Alfieri?

PASQUALE GALASSO. Qualche centinaio di persone.

PRESIDENTE. Ma quelli che facevano parte del direttivo erano anche killer?

PASQUALE GALASSO. Sì.

PRESIDENTE. Quindi, facevano una cosa e l'altra, non c'era una distinzione...

PASQUALE GALASSO. No, al di fuori di Alfieri, eravamo tutti killer.

PRESIDENTE. Che rapporti ci sono stati e ci sono con Cosa nostra?

PASQUALE GALASSO. Già dagli anni settanta, come le dicevo prima, Cosa nostra era presente principalmente a Napoli e in tutta la Campania con la famiglia Nuvoletta, che faceva capo a Liggio e poi dopo a Riina, Provenzano e Bagarella, con Zaza, che faceva capo ai Greco, poi con le famiglie Sciorio e Maisto.

PRESIDENTE. Che erano con Nuvoletta, Sciorio e Maisto?

PASQUALE GALASSO. I Maisto imparentati con i Nuvoletta. Sarebbe Enrico Maisto, che si è sposato la figlia di Antonio Orlando, zio di Lorenzo Nuvoletta. Queste erano le quattro famiglie mafiose che speculavano e sfruttavano... speculavano, con la forza dei mafiosi dietro le spalle, un po' tutta la criminalità campana. Cioè, voglio dire, Nuvoletta sfruttava questa forza... 
PRESIDENTE. Può spiegare alla Commissione che vuol dire che sfruttava questa forza del gruppo dei corleonesi?

PASQUALE GALASSO. All'epoca i corleonesi - diciamo la mafia, Cosa nostra - era un gruppo affermato, faceva paura. Quindi, quando si sapeva che Nuvoletta o Zaza erano mafiosi, erano collegati a Cosa nostra, nessuno si permetteva di dargli fastidio e di aggredirli, finché non venne fuori Cutolo.

PRESIDENTE. Spieghi bene questa cosa.

PASQUALE GALASSO. Nel 1978-1979 evade Cutolo e comincia a creare un marasma a Napoli, incomincia ad imporre finanche a queste famiglie legate ai mafiosi le tangenti sui loro traffici illeciti.

PRESIDENTE. Cioè lui chiedeva un tot a cassa di sigarette, mi pare?

PASQUALE GALASSO. Non solo sulle casse di sigarette ma anche altri interessi. Cutolo mirava un po' a tutto. Quindi, in tutto questo contesto, si arriva che... dopo le rimostranze un po' di tutti i gruppi si arriva a quell'epoca che dicevo prima, nel 1980-1981, nella quale ci sono state numerose riunioni presso l'abitazione di Lorenzo Nuvoletta, dei Nuvoletta, in Marano, dove si discuteva, appunto, tra tutti questi gruppi criminali campani di dire a Cutolo, di imporre a Cutolo la tranquillità, di lasciarli perdere. I Nuvoletta si impegnarono loro a fare da mediatori ancora una volta per restare sulla scena criminale campana, per acquisire ancora importanza sulla scena criminale. Quindi, si arriva prima ad un'intesa di tregua, poi di pace, che Cutolo non mantiene. Prima ancora, a fine 1980, con l'omicidio di Francesco Fabbrocino, fratello di Mario Fabbrocino, e poi dopo...

PRESIDENTE. Commesso dai cutoliani?

PASQUALE GALASSO. Da Cutolo, come pure il ferimento, sempre in quell'epoca, dopo l'omicidio di Francesco Fabbrocino, di Mario Fabbrocino. 
PRESIDENTE. Se non ho capito male, c'erano queste quattro famiglie, Nuvoletta, Sciorio, Maisto, Orlando, che facevano riferimento al gruppo dei corleonesi?

PASQUALE GALASSO. Sì. Dalla fine degli anni sessanta fino all'epoca di Cutolo hanno un po' sfruttato questa loro affiliazione con i mafiosi.

PRESIDENTE. Cutolo, invece, cercava di avere lui il comando su tutta la Campania, su tutti gli affari?

PASQUALE GALASSO. No, Cutolo voleva spazio ma sempre, poi l'ho capito, con l'appoggio sottobanco dei mafiosi.

PRESIDENTE. Anche Cutolo?

PASQUALE GALASSO. Sì.

PRESIDENTE. E di quali mafiosi?

PASQUALE GALASSO. Credo principalmente i corleonesi, facendo capo a Liggio, lui Cutolo credo che nel carcere che era ben presente...

PRESIDENTE. I corleonesi quindi appoggiavano tanto Nuvoletta quanto Cutolo?

PASQUALE GALASSO. Sì, come pure Cutolo... Si arriva ad un certo punto che i referenti politici di Nuvoletta sono pure di Cutolo, come poi i referenti politici di Cutolo diventano di Alfieri, subito dopo. Signor presidente, ci sono politici e malavitosi, come Nuvoletta, che pur di rimanere sulla scena cambiano amico da un momento all'altro, da un'epoca all'altra.

PRESIDENTE. Ora arriveremo a questo. La cosa che non ho molto chiara è che queste quattro famiglie si appoggiano ai corleonesi ed essendo appoggiate dai corleonesi... 
PASQUALE GALASSO. Mah, corleonesi diciamo a Cosa nostra, Greco, Bontate...

PRESIDENTE. Anche a Bontate, a tutti, insomma?

PASQUALE GALASSO. Sì, a Cosa nostra.

PRESIDENTE. Poi Cosa nostra ad un certo punto appoggerebbe non solo questi quattro ma anche Cutolo?

PASQUALE GALASSO. Lo dimostra il fatto che ho avuto contezza, durante le riunioni fatte da tutti i gruppi criminali campani presso l'abitazione dei Nuvoletta in Marano...

PRESIDENTE. Quella del 1981?

PASQUALE GALASSO. Sì, del 1981, dove Nuvoletta prese una posizione che non era netta come quella del suo braccio armato, di Bardellino, che rimproverava a Cutolo, a Pasquale Cutolo, a Casillo, che non dovevano fare abusi, che dovevano lasciare in pace gli altri gruppi malavitosi. Ma glielo diceva con schiettezza, con franchezza. Invece Nuvoletta, quello che hanno fatto sempre, con la loro ambiguità, facevano il doppio gioco. Alla fine, si capì e questo si è capito con l'omicidio di Salvatore Alfieri e con quello di mio fratello, dove i Nuvoletta si staccarono definitivamente da Alfieri e, diciamo, da me. Normalmente si danno le condoglianze quando muore un familiare, invece Nuvoletta non venne né da Alfieri né da me, da nessuno, non ci mandò condoglianze.

PRESIDENTE. Era un segnale?

PASQUALE GALASSO. Benissimo. Dimostrò che lui condivideva, stava più con Cutolo che con noi, perché in quel momento Nuvoletta ci vedeva perdenti. E' un po' la mentalità dei politici, di alcuni politici campani: se vedono uno perdente lo scaricano 
e vanno a creare l'amicizia con il camorrista vincente della zona, rapporti pure normali, formali, ma comunque un rapporto tra un camorrista ed un politico. Questa è la figura di Nuvoletta, perché i Nuvoletta nascono ambigui. Forse non so se contattando qualche politico hanno preso questa mentalità oppure ce l'hanno in loro stessi.

PRESIDENTE. Nuvoletta quindi faceva capo a queste persone, che lei ha detto, di Cosa nostra. Bardellino, che lei dice uomo più rigoroso, più rigido, nel confronto con Cutolo?

PASQUALE GALASSO. Bardellino era un malavitoso che apprezzavo molto perché era franco, non amava sotterfugi, ambiguità.

PRESIDENTE. Faceva riferimento anche lui a gruppi mafiosi?

PASQUALE GALASSO. Bardellino negli anni settanta era il braccio armato di Nuvoletta. I Nuvoletta hanno sfruttato molto la ferocia di Bardellino, grosso killer, e quindi sono andati avanti per dieci anni.

PRESIDENTE. Bardellino faceva riferimento agli stessi mafiosi cui faceva riferimento Nuvoletta?

PASQUALE GALASSO. Sì, so principalmente a Cosa nostra. Bardellino conosceva, so per bocca sua, Buscetta e tutti gli altri di Cosa nostra.

PRESIDENTE. Conosceva anche Bontate?

PASQUALE GALASSO. Sì.

PRESIDENTE. E gli altri? 
PASQUALE GALASSO. Diciamo che Bontate aveva uno stretto legame pure con Peppino Sciorio, della famiglia Sciorio.

PRESIDENTE. Una delle quattro famiglie di cui parlava prima. I rapporti con la 'ndrangheta?

PASQUALE GALASSO. Sono rapporti episodici, di tanto in tanto. Ci sono rapporti.

PRESIDENTE. Legati a traffici specifici?

PASQUALE GALASSO. No, diciamo all'amicizia, allo scambio di favori e al traffico di armi, principalmente.

PRESIDENTE. Può spiegare?

PASQUALE GALASSO. Almeno nel nostro gruppo tenevamo rapporti con un certo Muto.

PRESIDENTE. Il quale?

PASQUALE GALASSO. Con il quale avevamo rapporti di amicizia, io non lo conosco ma aveva rapporti di amicizia con Alfieri e con altri nostri associati, quali Maiale, della zona salernitana, Mario Pepe...

PRESIDENTE. Anche Mario Pepe era di Salerno?

PASQUALE GALASSO. Sì, dell'agro nocerino-sarnese ma è uno che viene dopo che si è formata la nostra organizzazione.

PRESIDENTE. Può spiegare questa questione del traffico delle armi come affare attorno al quale c'è un rapporto con questo gruppo di 'ndrangheta? 
PASQUALE GALASSO. Almeno con questo Muto c'era un rapporto di amicizia, di reciproci favori. Muto è un altro che si interessa di appalti, almeno in quella zona iniziale, scendendo giù, della Calabria. Quindi, per questi rapporti di appalti, con un altro imprenditore nostro associato lui si conosceva...

PRESIDENTE. Chi è questo imprenditore?

PASQUALE GALASSO. Luigi Romano.

PRESIDENTE. Sempre quello che ha detto prima?

PASQUALE GALASSO. Sì. Aveva intessuto rapporti inerenti agli appalti pubblici e quindi si crea questo rafforzamento di amicizia tra Muto e la nostra organizzazione. Ma diciamo che è un rapporto che poi mantengono nostri associati, quale Visciano Angelo, Maiale, Pecoraro.

PRESIDENTE. Lei conosce il Maisto di Praia a mare?

PASQUALE GALASSO. No.

PRESIDENTE. Mi pare, quindi, che mentre il rapporto con Cosa nostra è più fisso quello con la 'ndrangheta è diverso.

PASQUALE GALASSO. Sì, si andava a creare un rapporto sporadico, non con una certa intesa, una certa frequentazione: era un rapporto più lieve.

PRESIDENTE. Può indicare alcuni affari fatti insieme con la 'ndrangheta?

PASQUALE GALASSO. C'era il traffico di armi: talvolta ci fornivano le armi.

PAOLO CABRAS. Vi rifornivate di armi dalla 'ndrangheta? 
PASQUALE GALASSO. Sì; fornivano armi a questi gruppi camorristici e a noi associati.

PRESIDENTE. Dove prendeva le armi la 'ndrangheta?

PASQUALE GALASSO. Non so.

PRESIDENTE. Che tipo di armi erano?

PASQUALE GALASSO. Un po' di tutto.

PRESIDENTE. Anche esplosivo?

PASQUALE GALASSO. Sì, pure esplosivo.

PRESIDENTE. Armi corte e lunghe?

PASQUALE GALASSO. Sì.

PRESIDENTE. Vi erano rapporti anche con esponenti della Sacra corona unita?

PASQUALE GALASSO. Per la Sacra corona unita, fino a quando Cutolo non evade dal carcere, in Puglia di delinquenza organizzata non ce n'è; Cutolo è quello che da latitante si porta in Puglia e comincia a creare i primi associati malavitosi pugliesi alla NCO. Dopo Cutolo si va a creare questa associazione di gruppi delinquenziali pugliesi e ci sono rapporti fra questa associazione e le nostre associazioni campane.

PRESIDENTE. Può farci un po' di nomi?

PASQUALE GALASSO. Sì; dall'inizio degli anni ottanta fino agli ultimi tempi c'è un rapporto di traffico e contrabbando di sigarette e droga fra Gionta, i gruppi camorristici e i gruppi delinquenziali pugliesi. C'è il rapporto di un nostro gruppo 
camorristico, quello che fa capo a Visciano, a Di Martino e a Falanga Felice, sempre per traffici di contrabbando. C'è un rapporto di D'Alessandro Michele, del gruppo D'Alessandro di Castellammare, con altri associati pugliesi.

PRESIDENTE. Con quali?

PASQUALE GALASSO. Non so i nomi, ma so che D'Alessandro è ben presente in Puglia, dove hanno diverse abitazioni.

PRESIDENTE. In quale area della Puglia: nel Salento o nel foggiano?

PASQUALE GALASSO. Credo sia nel foggiano sia nel Salento.

PRESIDENTE. Lei non parla mai, per quanto riguarda gli affari, di traffico di stupefacenti: come mai, neanche le altre famiglie lo facevano?

PASQUALE GALASSO. Penso che, o meglio certamente, quasi tutti i gruppi camorristici campani si occupano di stupefacenti, ma diciamo che lo fanno di più quei gruppi camorristici che non sono presenti negli affari degli appalti pubblici.

PRESIDENTE. Quindi, quelli che non si occupano di appalti si occupano di stupefacenti?

PASQUALE GALASSO. Sì, invece principalmente il gruppo Contini, Mallardo, Licciardi si è interessato ultimamente, dopo la morte di Bardellino, degli appalti pubblici ma contestualmente ha sempre fatto traffico di stupefacenti. A noi ci risulta perché da un decennio abbiamo rapporti con Licciardi.

PRESIDENTE. Per stupefacenti intende eroina o cocaina?

PASQUALE GALASSO. Tutt'e due, ma pure le droghe leggere. 
PRESIDENTE. Come avviene questo traffico: loro curano l'importazione di grosse quantità oppure lo smercio sul territorio?

PASQUALE GALASSO. Diciamo tutt'e due le cose: curano l'importazione e pure lo smercio al minuto.

PRESIDENTE. Sa come e da dove si riforniscono?

PASQUALE GALASSO. No, le fonti non le conosco, ma diciamo con i loro referenti esteri, elementi camorristici che si sono trasferiti all'estero e si sono messi in contatto con altri elementi malavitosi esteri, creando questo traffico.

PRESIDENTE. Lei dice che il gruppo Alfieri si occupa di appalti a partire dal 1980, in sostanza dalla ricostruzione dopo il terremoto; è così?

PASQUALE GALASSO. Diciamo che il gruppo Alfieri cresce nel tempo dopo l'omicidio Casillo ed è presente fino ad arrivare totalmente a tutti gli appalti pubblici.

PRESIDENTE. Prima di occuparsi degli appalti, di che cosa si occupava?

PASQUALE GALASSO. Di altri traffici illeciti, come principalmente l'usura.

PRESIDENTE. Faceva estorsioni?

PASQUALE GALASSO. Estorsioni di meno, ma diciamo rapine e traffici di importazione.

PRESIDENTE. Importazione di che cosa?

PASQUALE GALASSO. Principalmente carni.

PRESIDENTE. Cioè? 
PASQUALE GALASSO. Importavano carne dall'estero e la vendevano nelle zone vesuviana e nolana.

PRESIDENTE. E questo non era lecito?

PASQUALE GALASSO. Era apparentemente legale ma si trattava di un reinvestimento dei profitti illeciti.

PRESIDENTE. Si occupava anche di contrabbando di sigarette e di tabacchi?

PASQUALE GALASSO. Forse negli anni settanta.

PRESIDENTE. Cosa nostra e quelli della mafia sono mai intervenuti per favorire la cessazione della guerra fra diverse organizzazioni della camorra? Sono mai intervenuti per cercare di mettere un po' di ordine in Campania?

PASQUALE GALASSO. Cosa nostra? L'intervento principale è durante le tensioni fra Cutolo e tutti i gruppi camorristici campani.

PRESIDENTE. Interviene Cosa nostra?

PASQUALE GALASSO. Interviene Cosa nostra dietro Nuvoletta e, diciamo, i loro rappresentanti mafiosi ma, ad un certo momento, a Cosa nostra interessava - credo certamente pure Cutolo, che nel 1981, specialmente dopo la vicenda Cirillo, nelle carceri era presente in un modo strepitoso e alto.

PRESIDENTE. Può spiegare alla Commissione qualcosa sulla riunione a Vallesana nel $1982 ?$

PASQUALE GALASSO. Sì; allora evade Cutolo dal manicomio di Aversa... 
PRESIDENTE. In che epoca siamo?

PASQUALE GALASSO. Nel 1978-79. Comincia quindi a creare tensioni un po' a tutti i gruppi camorristici campani e c'è una rivolta generale. Di queste lamentele si fanno carico i Nuvoletta, con a capo Lorenzo Nuvoletta, che riferisce tutto ai suoi capi mafiosi: ricordo che nel 1981 si facevano queste riunioni a Vallesana dove, per esplicita richiesta di Lorenzo Nuvoletta e Bardellino, io e Alfieri eravamo presenti (e talvolta qualche altro nostro associato). Su queste lamentele e su questa situazione i Nuvoletta volevano speculare ancora una volta e far capire a tutti i gruppi malavitosi campani che loro, comunque, erano i più forti, quelli che incidevano sulla pace, sulla tregua, sull'equilibrio criminale campano. Sono stato presente a diverse riunioni; ricordo che ad alcune di queste eravamo circa un centinaio, i rappresentanti di tutte le famiglie campane.

PRESIDENTE. Un centinaio di persone?

PASQUALE GALASSO. Sì.

PRESIDENTE. E nessuno si era accorto che eravate là?

PASQUALE GALASSO. No; in quel momento venni a sapere da Alfieri e Alfieri dallo stesso Nuvoletta che non c'erano problemi, neanche per quanto riguardava le forze dell'ordine che lui riusciva a controllare, riusciva a darci tranquillità. La nostra perplessità derivava dal pericolo che durante le nostre riunioni potessero intervenire i carabinieri facendo accadere un marasma. Nuvoletta invece ci ha sempre tranquillizzati e talvolta io e Alfieri abbiamo visto, scendendo da Vallesana, la masseria dei Nuvoletta, qualche auto dei carabinieri appena fuori dell'abitazione di Nuvoletta. Quella per noi era la dimostrazione che Nuvoletta era ben protetto. Ricordo che all'epoca Nuvoletta era in stretto rapporto con un politico nazionale di grosso rilievo.

PRESIDENTE. Chi era? 
PASQUALE GALASSO. Gava. Questo perché se ne parlava durante le riunioni; talvolta io, Alfieri e qualche altro componente della sua organizzazione abbiamo pranzato con Lorenzo Nuvoletta su esplicita sua richiesta. Quindi se ne parlava perché vedevamo un'ostentata tranquillità a casa di Lorenzo Nuvoletta mentre a quell'epoca anche l'abitazione dell'ultimo malavitoso era soggetta a perquisizione.

PRESIDENTE. Nuvoletta non subiva perquisizioni?

PASQUALE GALASSO. No, almeno per quanto mi consta; quegli appuntamenti, quelle riunioni così sfacciate...

PRESIDENTE. Con quale mezzo vi andavate? Con le vostre auto?

PASQUALE GALASSO. Sì, con le nostre auto.

PRESIDENTE. Si trattava, dunque, di 70-80 automobili!

PASQUALE GALASSO. Sì.

PRESIDENTE. Dove le mettevate?

PASQUALE GALASSO. La masseria di Nuvoletta ha un piazzale molto grande, quindi le automobili potevano essere messe sia nella strada che portava alla masseria sia all'interno della masseria stessa, nel grande piazzale dove potevano essere parcheggiate benissimo 50-100 automobili.

PRESIDENTE. Quante riunioni di questo tipo ci sono state?

PASQUALE GALASSO. Ricordo particolarmente una delle ultime riunioni dell'estate 1981 nel corso della quale dovevamo decidere di chiarirci una volta per sempre le tensioni che ci venivano create dai cutoliani. Ricordo che in quel momento erano 
presenti nella masseria di Nuvoletta Riina, Provenzano e Bagarella. Erano questi i nomi.

PRESIDENTE. Erano con voi o in un altro posto?

PASQUALE GALASSO. Erano a circa 50-100 metri da noi, in un villino in un castagneto poco lontano da noi.

PRESIDENTE. Sempre nella proprietà di Nuvoletta?

PASQUALE GALASSO. Sì, perché durante queste riunioni si creavano tensioni tra alcuni componenti della riunione; maggiormente si creò una tensione particolare tra Antonio Spavone, il famoso o' malommo, e gli Zaza e ci fu un battibecco tra di loro. Spavone venne sparato nel 1976 dagli Zaza; Spavone ritenne opportuno in quella riunione far presente che non c'era solo il pazzo Cutolo che dava fastidio a tutti gli altri gruppi camorristi ma anche altre famiglie camorriste, come gli Zaza.

PRESIDENTE. Spavone era rimasto solo ferito?

PASQUALE GALASSO. Sì. Era il 1976 e Spavone sapeva che erano stati gli Zaza.

PRESIDENTE. Come facevano ad influire Riina e gli altri siciliani che stavano separati?

PASQUALE GALASSO. Durante queste ed altre tensioni ci siamo accorti, io e qualche altro mio amico, che Lorenzo Nuvoletta, Michele Zaza e qualche altro partecipante a queste riunioni chiedevano il permesso di allontanarsi un momento e ritornavano dopo mezz'ora o un'ora portando nuove notizie. A volte Lorenzo Nuvoletta diceva come bisognava fare, che cosa lo invitava a fare; silenziosamente vedevamo che anche i componenti cutoliani assimilavano quello che diceva Nuvoletta. D'altronde si sapeva già, per esplicita ammissione di Lorenzo Nuvoletta, che i siciliani erano nella sua proprietà. 
MARIO CLEMENTE MASTELLA. La protezione cui lei ha fatto cenno era di Gava o di uomini di Gava?

PRESIDENTE. Si tratta di un aspetto molto delicato che interessa molto la Commissione e su cui in seguito le verranno rivolte domande specifiche. L'onorevole Mastella le chiede se tale protezione venisse direttamente dal senatore Gava o se fosse di uomini di Gava. Quali sono gli elementi che Nuvoletta vi dava per farvi intendere questo?

PASQUALE GALASSO. Per quanto mi consta, la protezione... A me consta il rapporto tra Gava e Nuvoletta.

PRESIDENTE. Da cosa le consta tale rapporto?

PASQUALE GALASSO. Sia dall'ammissione di Lorenzo Nuvoletta sia da quanto durante i pranzi che eravamo soliti fare nell'abitazione di Lorenzo Nuvoletta veniva detto da altri suoi associati e persone di fiducia che appartenevano al suo direttivo, come Salvatore Normale, il quale all'epoca ci faceva ben presente che giorni prima si erano visti con Gava. Questo è il rapporto tra Nuvoletta e Gava e certamente i Nuvoletta godevano degli appoggi dei referenti di Gava in zona, come d'altronde ho toccato con mano, Alfieri ed io godevamo dei favori dei referenti di Gava nella nostra zona.

PRESIDENTE. Con quali personalità politiche ha parlato direttamente?

PASQUALE GALASSO. Noi? Io?

PRESIDENTE. Sì, lei.

PASQUALE GALASSO. Ho parlato con i maggiori referenti di Gava nella nostra zona, per esempio Riccio, presidente della USL e sindaco di San Paolo Belsito, il senatore 
Meo, Giuseppe D'Antuono, Pasquale Catapano, presidente della USL e fedelissimo di Gava da sempre, fin dalle mani di suo padre Ciccio Catapano. Ci sono Ciccio Liguori, Achille Marciano...

PRESIDENTE. Di dov'è Ciccio Liguori?

PASQUALE GALASSO. Di Poggiomarino.

PAOLO CABRAS. Era il sindaco di Poggiomarino, era la sua città.

PASQUALE GALASSO. Ha fatto il sindaco per un ventennio. Poi Achille Marciano e tutti gli altri; a Pompei Maghetti e Tucci e tanti altri ancora.

PRESIDENTE. Gli altri con chi avevano rapporti?

PASQUALE GALASSO. Quali altri?

PRESIDENTE. Quelli del clan Alfieri o del clan Nuvoletta con quali uomini politici avevano rapporti, che lei sappia?

PASQUALE GALASSO. Posso parlarvi della zona nolana, vesuviana e sorrentina...

PRESIDENTE. Quella che lei conosce.

PASQUALE GALASSO. Ma della zona a nord di Napoli non posso darvi spiegazioni o fare nomi perché non ho avuto contatti.

PRESIDENTE. Lei ha fatto un elenco di persone aventi responsabilità politiche che lei ha contattato direttamente. A questo punto la domanda è la seguente: gli altri, cioè Nuvoletta o Alfieri, con quali uomini politici avevano rapporti in quella zona? 
PASQUALE GALASSO. Per quanto mi consta, il politico con il quale tutti questi grossi malavitosi, quali Nuvoletta, Alfieri, Cutolo e pochi altri, hanno avuto sempre contatti vuoi direttamente o vuoi per suoi referenti è sempre stato Antonio Gava, diciamo la corrente dorotea facente capo a Gava.

PRESIDENTE. Facciamo un passo indietro: nelle riunioni a Vallesana chi erano i rappresentanti di Cutolo? Cutolo veniva direttamente o mandava qualcuno?

PASQUALE GALASSO. No, Cutolo era già stato arrestato; veniva Pasquale Cutolo...

PRESIDENTE. Che è il fratello.

PASQUALE GALASSO. Vincenzo Casillo e il direttivo di Cutolo; in tutto 10-15 persone.

PRESIDENTE. Casillo era un uomo molto vicino a Cutolo?

PASQUALE GALASSO. Sì.

PRESIDENTE. E' quello che sarà ucciso?

PASQUALE GALASSO. Sì.

PRESIDENTE. Circa le strutture, avete rapporti...

MARIO CLEMENTE MASTELLA. Vorrei rivolgere un'altra domanda.

ANTONIO BARGONE. Tutti dobbiamo rivolgere domande ma possiamo farlo dopo.

MARIO CLEMENTE MASTELLA. Se ci sono connivenze, vuol dire che anche polizia e carabinieri partecipavano alle riunioni? 
PRESIDENTE. E' la prima volta che lei, onorevole Mastella, partecipa ad un'audizione di un collaboratore di giustizia. Se lei prende appunti, onorevole Mastella, dopo potrà porre tutte le questioni che riterrà opportune. Chiedevo, dunque, se le risultino rapporti con le organizzazioni straniere.

PASQUALE GALASSO. Prima vorrei rispondere ancora all'altra domanda. Un'altra persona di fiducia, nelle mani sia di Antonio Gava, della corrente dorotea e di Alfieri, è il generale De Sena. Signor presidente, questo è ormai così sfacciato, chiaro e trasparente, che mi chiedo come voi - dico "voi" per dire lo Stato italiano - facciate a non accorgervi di certi rapporti.

PAOLO CABRAS. Quali rapporti?

PASQUALE GALASSO. Dei rapporti tra questi amministratori collusi sia con l'ambiente malavitoso, sia con i loro capi referenti politici. E' una cosa che ormai sanno tutti, almeno i cittadini miei compaesani.

PRESIDENTE. Va bene. Quindi, lei dice che un altro dei nomi...

PASQUALE GALASSO. I cittadini nolani e vesuviani lo sanno tutti. Hanno sempre saputo di un rapporto tra Alfieri ed il generale De Sena, sindaco di Nola.

PRESIDENTE. Sempre seguendo il filo logico fondamentale della struttura della camorra, anche se ogni tanto andiamo da qualche altra parte, che può dirci a proposito della presenza delle organizzazioni camorristiche sul territorio nazionale? Per esempio, sono presenti nel Lazio ed a Roma?

PASQUALE GALASSO. Sì, dopo l'omicidio Casillo a Roma tenevamo i nostri referenti. Sono rimasti i coniugi Cillari e maggiormente Ciro Maresca, Nicoletti e, ultimamente, Sepe Marzio e Cesarano. Ma principalmente i coniugi Cillari, Ciro Maresca e Nicoletti. Dopo l'omicidio Casillo, Ciro Maresca si trasferisce a Roma, dove vive da 10 anni. Qui 
fa i traffici illeciti più svariati. Di qualsiasi cosa la nostra organizzazione aveva bisogno malavitosamente, faceva riferimento a Ciro Maresca.

PRESIDENTE. In altre parti del Lazio è presente?

PASQUALE GALASSO. Sono presenti in altre zone del Lazio.

PRESIDENTE. Nella zona di Latina, per esempio? Al Sud? Può spiegare con quali persone?

PASQUALE GALASSO. Inizialmente c'erano i Maiulo, i Moccia, i Bardellino. Fino al 1988 c'erano i Bardellino e il clan casalese, che si espandeva fino a Latina. Quindi, quando avevamo bisogno di qualcosa, ci rivolgevamo a Bardellino, il quale sistemava tutto o ci metteva in contatto con le persone con le quali dovevamo...

PRESIDENTE. E dopo il 1988?

PASQUALE GALASSO. Dopo ci sono rimasti i casalesi, mentre i Maiulo sono un po' scomparsi, perché nel frattempo è morto uno dei fratelli. Principalmente, i casalesi.

PRESIDENTE. E in altre aree del Lazio?

PASQUALE GALASSO. Signor presidente, ci sono elementi camorristici che fanno riferimento alla nostra organizzazione e ad altre organizzazioni camorristiche, ma non sono di una certa rilevanza. Anche perché sono zone tranquille, ci si va per fare un traffico o un reinvestimento apparentemente legale. Quindi, si sta abbastanza tranquilli.

PRESIDENTE. Quali sono gli interessi di Alfieri e di Ruocco a Latina?

PASQUALE GALASSO. Durante la guerra di Cutolo e dopo l'omicidio Casillo, Peppino Ruocco, che apparteneva ad una famiglia di commercianti ortofrutticoli (da sempre 
presente sul territorio di Latina per il commercio di questi prodotti, ma in maniera modesta), divenuto figura emergente porta la famiglia ad estendere il suo potere, su tutti i prodotti ortofrutticoli, in quasi tutta la zona di Latina. Da sempre c'è una presenza malavitosa, per un certo periodo collegata a Cutolo da rapporti amicali, che poi passa nelle mani di Alfieri e di Pasquale Ambrosino. Quest'ultimo ha un'industria in zona per l'importazione e l'esportazione di prodotti orticoli esotici e no, perfino dalla Cina. Quindi, nel mercato ortofrutticolo c'è la presenza della malavita. Ultimamente, quasi tutto era nelle mani di Peppino Ruocco, ma ci sono sempre state influenze siciliane. Talvolta Peppino Ruocco riesce pure a mortificare elementi appartenenti a Cosa nostra.

PRESIDENTE. E' molto forte questo Ruocco!

PASQUALE GALASSO. E' morto. Lo abbiamo ammazzato noi stessi.

PRESIDENTE. E nella zona di Fondi?

PASQUALE GALASSO. C'è la presenza di elementi della 'ndrangheta, c'è l'influenza dei casalesi (principalmente, un periodo è stato dei Maiulo e dei Moccia). A Fondi sono presenti i figli di Mico Tripodo, ammazzato nel carcere di Poggioreale da Cutolo, dietro compenso di Paolo De Stefano.

PRESIDENTE. Cioè?

PASQUALE GALASSO. Paolo De Stefano era calabrese, aveva una faida con i Tripodo. Il capo dei Tripodo era don Mico. Mi sono trovato là quando Cutolo l'ha fatto ammazzare in carcere, nel 1976. L'ho soccorso io don Mico Tripodo. Cutolo mi riferì dei suoi rapporti con De Stefano e che era stato lui a ordinare quell'omicidio.

PRESIDENTE. E come avvenne questo omicidio in carcere? 
PASQUALE GALASSO. Lo compì Agrippino Effice, un associato NCO, un affiliato di Cutolo. Ricordo bene che Cutolo, che stava a Poggioreale, organizzò il tutto. Ricordo che un mese prima si fece trasferire in un padiglione (credo fosse il padiglione Milano), dove vi rimase. Furono Agrippino Effice e, mi sembra, Domenico Morelli, di Sant'Antimo, quelli che materialmente hanno ammazzato don Mico Tripodo, che si trovava di fronte alla mia cella.

PRESIDENTE. Ma come l'ammazzarono?

PASQUALE GALASSO. A coltellate. Ricordo che lo soccorsi io don Mico Tripodo.

PRESIDENTE. L'omicidio del Ruocco da cosa fu provocato?

PASQUALE GALASSO. A Fondi ci sono i figli di Tripodo...

PRESIDENTE. Ah, sì, stava dicendo dei figli di Tripodo.

PASQUALE GALASSO. Ci sono i figli di Tripodo ma con l'influenza dei Casalesi.

PRESIDENTE. Diceva prima che Ruocco è stato ucciso. Per quale ragione ciò è accaduto?

PASQUALE GALASSO. Giuseppe Ruocco è di Piazzola di Nola, il paese di Carmine Alfieri. Ultimamente, Giuseppe Ruocco gestiva un po' tutti gli affari di Carmine Alfieri e quasi tutti i suoi rapporti politici ed istituzionali. Da diversi anni, Carmine Alfieri temeva Giuseppe Ruocco per la sua ferocia di grosso killer. Quasi tutti i morti, nella guerra contro Cutolo e altri gruppi camorristici, storicamente contro gli Alfieri... è stato Giuseppe Ruocco quello che, materialmente, ha vendicato la morte di Salvatore Alfieri. Quindi, diviene una figura emergente; era un giovane che amava la bella vita e che aveva un buon dialogo con tutti gli ambienti sociali delle zone del nolano e del vesuviano. Questo accrebbe la tensione e la paura in Carmine Alfieri, il quale temeva di fare la stessa fine di Bardellino. 
PRESIDENTE. Quindi, fu Carmine Alfieri a ordinare l'omicidio...

PASQUALE GALASSO. Sì. Carmine Alfieri porta sul tavolo del nostro direttivo, una prima volta un paio d'anni prima, le lamentele contro Giuseppe Ruocco e la sua decisione di ammazzarlo. Soprattutto io, e poi qualche altro componente del nostro direttivo, facemmo leva affinché non succedesse questa atrocità. Questo è un episodio che mi ha fatto toccare con mano le belve e la melma di questa mia vita criminale. Forse pure questo ha inciso a farmi cambiare vita.

PRESIDENTE. Lei si oppose, però?

PASQUALE GALASSO. Mi opposi, io e qualche altro componente e quindi Alfieri non decise.

PRESIDENTE. In che periodo?

PASQUALE GALASSO. Nel 1989.

PRESIDENTE. Quindi, Alfieri non decise l'omicidio?

PASQUALE GALASSO. No, perché noi dicemmo apertamente che se si incominciava ad ammazzare, se ammazzavamo Giuseppe Ruocco, che per noi era un capo storico, certamente si sarebbero create tensioni in tutti gli altri gruppi nostri associati e pure in noi stessi. Quindi, era una cosa non giusta, non fatta per bene. Ci si poteva parlare con Peppino Ruocco ed eventualmente farlo correggere sul suo carattere, sul suo comportamento ma Carmine Alfieri ritenne opportuno di non parlarne proprio. Noi lo invitammo a creare un dialogo con lo stesso Giuseppe Ruocco, che poi era suo figlioccio, cresimato pure, Carmine Alfieri l'aveva pure cresimato. In quel momento si superò questa...

PRESIDENTE. Poi dopo come si arriva all'omicidio? 
PASQUALE GALASSO. Poi dopo... Carmine Alfieri ha avuto sempre questa idea, perché si sentiva esposto, dato che Giuseppe Ruocco era del suo stesso paese, della sua stessa zona. Vedeva che Giuseppe Ruocco cresceva sempre di più, aveva rapporti con varie autorità istituzionali, nostri complici, nostri collusi e quindi alla fine, per l'ennesima volta, Carmine Alfieri riportò sul tavolo questa decisione. Credo, almeno da parte mia, mio malgrado e forse pure per qualche altro componente del direttivo, aderimmo a questa decisione atroce.

PRESIDENTE. Come mai aderiste, vi convinceste?

PASQUALE GALASSO. Perché non si poteva fare altrimenti. Signor presidente, almeno io non riuscivo a trovare un altro... Pensai pure bene eventualmente di avvertire lo stesso Giuseppe Ruocco ma alla fine poteva solamente soccombere: né Giuseppe Ruocco mi credeva né eventualmente tra Giuseppe Ruocco e Carmine Alfieri si andava ad un chiarimento. Poteva solamente soccombere. Questa è la legge, purtroppo, della malavita, atroce ed immonda.

PRESIDENTE. In quali altre regioni siete presenti? In Toscana?

PASQUALE GALASSO. Sì.

PRESIDENTE. In quali zone?

PASQUALE GALASSO. Diciamo in quasi tutta la Toscana.

PRESIDENTE. Umbria?

PASQUALE GALASSO. Poco, almeno per quanto riguarda la nostra organizzazione, no.

PRESIDENTE. Emilia Romagna? 
PASQUALE GALASSO. Sì.

PRESIDENTE. In Romagna o in Emilia, cioè sulla costa o nell'interno?

PASQUALE GALASSO. Sulla costa.

PRESIDENTE. Che attività si svolgono?

PASQUALE GALASSO. Ci sono associati di nostri gruppi camorristici che sono presenti in zona e si investe, si specula su diversi canali.

PRESIDENTE. Veneto?

PASQUALE GALASSO. No.

PRESIDENTE. Lombardia?

PASQUALE GALASSO. Sì.

PRESIDENTE. Liguria?

PASQUALE GALASSO. Sì, principalmente a Genova ci sono i mafiosi con GiontaNuvoletta.

PRESIDENTE. Piemonte?

PASQUALE GALASSO. Poco, almeno dalla nostra organizzazione. C'era...

PRESIDENTE. Abruzzo? 
PASQUALE GALASSO. No. C'era in Piemonte quel rapporto nostro con elementi mafiosi.

PRESIDENTE. Quello per il congegno per Casillo?

PASQUALE GALASSO. Benissimo. Quelli che poi ci fornirono il congegno ma credo che oggi sono scomparsi.

PRESIDENTE. Era un mafioso?

PASQUALE GALASSO. Era un mafioso. Quindi, rapporti tra noi associazione camorristica e questi elementi mafiosi.

PRESIDENTE. In Abruzzo invece non ci sono vostre presenze?

PASQUALE GALASSO. No.

ROMEO RICCIUTI. Maisto si è rifugiato lì per tanti anni.

PASQUALE GALASSO. Ho capito ma non fa parte del mio gruppo.

PRESIDENTE. La domanda che la Commissione le fa non riguarda soltanto il suo gruppo, riguarda, per quello che lei sa, anche altri gruppi.

PASQUALE GALASSO. Posso dire in linea generale che in quasi tutta Italia ci sono elementi camorristici presenti, poi bisogna vedere la rilevanza o l'importanza di questi e quindi le difficoltà sociali che possono creare. Se è un piccolo malavitoso non può portare grossi problemi, quale può succedere...

ALFREDO GALASSO. Potrebbe approfondire questa domanda, chiedere quali sono gli affari... 
PRESIDENTE. Sì, ora spieghiamo. Noi stiamo lavorando sulla presenza delle varie organizzazioni mafiose nelle diverse regioni italiane, anche quelle, come dire, non tradizionali, dove c'è un insediamento nuovo. Allora, alla Commissione interessa sapere, oltre quello che lei ci ha detto, anche, se possibile, nelle singole aree, per quello che lei sa, che tipo di affari si fanno: si fa traffico di stupefacenti o si investe in affari legali, se si investe in affari legali in che tipo di affari. Questo perché abbiamo bisogno di capire bene come sono presenti le diverse organizzazioni mafiose su tutto il territorio nazionale e che tipo di affari fanno. Questa è la nostra esigenza. Ora, sulla base di quel che lei sa e può dire, ci risponda.

MARIO BORGHEZIO. Può precisare in Lombardia in quali zone?

PRESIDENTE. Può rispondere?

PASQUALE GALASSO. Almeno per quanto mi consta, in Lombardia, a Varese, poi Milano e tutto il circondario di Milano, principalmente.

PRESIDENTE. Si parla del gruppo Alfieri o in generale?

PASQUALE GALASSO. Principalmente, forse parlo in linea generale. Per il gruppo Alfieri, Milano, Varese, queste sono le zone.

PRESIDENTE. Può dire per cortesia nelle singole aree, se lo sa, che tipo di affari specifici si fanno?

PASQUALE GALASSO. Sono i soliti affari. Oltre a quello del traffico di stupefacenti sono ben individuati i gruppi che fanno questo traffico - sono quegli affari dovunque si può investire, si può trafficare, non so: dalle discoteche alle situazioni fallimentari.

PRESIDENTE. Cioè, acquistare aziende in fallimento? 
PASQUALE GALASSO. Sì. Non ultimo, per volontà di Alfieri, io, Alfieri ed altri nostri associati abbiamo fatto un investimento a Forlì, in Romagna. Rilevammo da una situazione fallimentare...

PRESIDENTE. Può spiegare meglio?

PASQUALE GALASSO. Sì. Rilevammo tre o quattro industrie che poi abbiamo venduto.

PRESIDENTE. Si ricorda quali industrie?

PASQUALE GALASSO. Sì.

PRESIDENTE. Le può dire?

PASQUALE GALASSO. I nomi non li ricordo ma sono industrie di avicoltura, polli e galline. Ci sono due o tre industrie grosse. Noi le rilevammo per fare una cortesia ad un amico di Alfieri. Ci siamo preoccupati di gestire un po' tutta questa situazione fallimentare per poi portarla a termine e alla fine, pure con i profitti, le abbiamo cedute allo stesso amico di Alfieri, che si trovava in una situazione fallimentare. Questo amico lo aveva aiutato a trovare un'amicizia con i giurati durante il processo per la strage di Torre Annunziata.

PRESIDENTE. Questo amico?

PASQUALE GALASSO. Di Alfieri. Quindi, quello per noi è stato un intervento isolato.

PRESIDENTE. Come si chiama questa persona di Forlì?

PASQUALE GALASSO. Silvio Balletta. 
PRESIDENTE. Che aveva aiutato Alfieri ad avere un contatto con i giurati del processo per la strage di Torre Annunziata?

PASQUALE GALASSO. Sì. Alfieri, per riconoscenza, per sdebitarsi, mi interpellò, per quanto è di mia conoscenza, per la mia managerialità imprenditoriale, per aiutare questo signore.

PRESIDENTE. Quindi, compraste queste aziende?

PASQUALE GALASSO. No, sistemammo, intervenimmo con soldi, con circa 1 miliardo e mezzo, due miliardi di lire. Sistemammo tutta la situazione prefallimentare di queste aziende e poi dopo le abbiamo cedute allo stesso Silvio Balletta, dandoci soldi e regolamento per quel che avevamo sborsato, con utili pure.

PRESIDENTE. Come faceste? Interveniste attraverso un commercialista, o direttamente?

PASQUALE GALASSO. Sempre in Romagna, a Forlì, era presente il gruppo di Mario Pepe per quanto riguarda l'usura principalmente, le situazioni prefallimentari e i supermercati.

PRESIDENTE. Avete rapporti per quanto riguarda le aste giudiziarie? Cercate di controllarle?

PASQUALE GALASSO. Questo è un altro punto importante: da sempre, forse già dagli anni settanta, i vari rapporti politico-camorristici consistevano in scambi di favori, in consulenze e aiuti politici per avvicinare talvolta il curatore, o il giudice, e mettere a disposizione del rappresentante camorrista della zona a loro referente questo tipo di favore. Mi ricordo che quello delle aste fallimentari è stato sempre uno dei profitti illeciti. 
PRESIDENTE. Spieghi meglio alla Commissione quanto avviene. Innanzitutto, in quali città succede?

PASQUALE GALASSO. A Napoli e a Roma, principalmente. Già negli anni settanta, sia a Napoli sia a Roma, il gruppo di Alfonso Rosanova, il padre spirituale di Cutolo, grosso camorrista anche lui legato alla corrente dorotea, curava tutte le aste fallimentari del tribunale di Napoli e di Roma.

PRESIDENTE. In che modo?

PASQUALE GALASSO. Non so... Loro erano aggiornati dalle loro amicizie dei vari immobili prefallimentari o riferibili a fallimenti già dichiarati: se era una cosa che interessava, perché potevano speculare, cominciavano a tessere tutto un piano e quindi si arrivava, o con le compiacenze di qualche avvocato, o con i loro referenti politici, al curatore e poi al giudice fallimentare. Si curava, nel tempo di qualche anno, tutta la vicenda delle aste pubbliche, finché non si portava quel bene ad un determinato prezzo e poi lo acquisivano.

PRESIDENTE. Ad un prezzo basso?

PASQUALE GALASSO. Sì.

PRESIDENTE. Quindi, la tecnica era questa: si seguiva la cosa, si cercava di ottenere il prezzo più basso possibile e poi si comprava?

PASQUALE GALASSO. Sì, oppure si mettevano direttamente in contatto con i falliti, tramite il curatore, e concordavano con tutti i creditori un prezzo misero, basso: rilevavano quindi il tutto, facendo talvolta qualche regalo, oppure dando una percentuale, ai falliti. Poi da tutti questi meccanismi possono uscire tante altre soluzioni strada facendo.

PRESIDENTE. Questo controllo a Napoli e a Roma continua adesso? 
PASQUALE GALASSO. Sì, continua adesso; almeno i nostri associati su Roma hanno ultimamente fatto dei grossi business con le proprietà di Caltagirone, principalmente in via Cortina d'Ampezzo e in via Courmayer: hanno rilevato interi palazzi del fallimento Caltagirone. Questo l'hanno gestito principalmente i coniugi Cillari su miei finanziamenti, dovuti sempre...

PRESIDENTE. Caltagirone è partecipe? Sa di questo?

PASQUALE GALASSO. Non mi risulta. Io solamente per un fattore di riconoscenza verso i Cillari, che hanno partecipato all'omicidio, perché ci hanno consentito di prendere Casillo e di dare una botta finale a Cutolo, li ho aiutati.

PRESIDENTE. Ci sono a Roma magistrati collusi che sono d'accordo con Cillari?

PASQUALE GALASSO. Sì.

PRESIDENTE. Chi sono?

PASQUALE GALASSO. Questo è coperto dal segreto istruttorio e preferirei non rispondere. Ne ho già parlato con i giudici competenti.

PRESIDENTE. Quali sono i giudici competenti per questa materia?

PASQUALE GALASSO. Oltre ai giudici della procura di Napoli e Salerno, quelli di Roma.

PRESIDENTE. Quanti sono? Questo lo può dire.

PASQUALE GALASSO. Cillari mi presentò direttamente una di queste persone.

PRESIDENTE. Ce ne sono anche altri? 
PASQUALE GALASSO. Io credo, per quanto mi risulta dal 1970 in poi, e da tutte le vicende fallimentari di cui sono a conoscenza, principalmente di Rosanova, Cillari, Maresca, che c'è una intesa con diversi elementi.

PRESIDENTE. Con diversi magistrati?

PASQUALE GALASSO. Oltre che magistrati, credo anche una cerchia di professionisti.

PRESIDENTE. Per esempio, cancellieri e tutto un giro nei tribunali?

PASQUALE GALASSO. Sì, qualche cancelliere compiacente e avvocati che vivono di questo. A Roma c'è un forte condizionamento ed un grosso intreccio, almeno per le aste giudiziarie.

PRESIDENTE. E a Napoli?

PASQUALE GALASSO. A Napoli è da sempre, almeno per quanto mi risulta: quando interessa una proprietà, ci mette sopra gli occhi il camorrista, che talvolta si scontra pure con qualche elemento politico, e poi si arriva ad un patto, o a un accordo.

PRESIDENTE. E può fare i nomi dei magistrati collusi a Napoli, o indicare la dimensione del fenomeno?

PASQUALE GALASSO. Per le aste fallimentari, non li so. Non ho mai frequentato le aste fallimentari per esplicito consiglio di mio padre.

PRESIDENTE. Però, a Roma sì?

PASQUALE GALASSO. Per Roma ho saputo queste cose tramite Cillari, come ho saputo quando Pino Cillari era socio di Rosanova, già negli anni settanta, e seguivano 
le aste: hanno fatto più di un investimento, rilevando opifici ed interi stabili, in via Veneto ed anche nella zona di Latina.

PRESIDENTE. Proprio a Latina o in paesi vicini?

PASQUALE GALASSO. Credo in provincia di Latina. Questi erano rapporti fra Cillari e Rosanova: all'epoca, negli anni settanta, Rosanova seguiva principalmente le aste fallimentari.

PRESIDENTE. Voi avete rapporti con organizzazioni criminali di altri paesi, o ci sono vostre diramazioni, per esempio, in Germania, Francia, Inghilterra?

PASQUALE GALASSO. Qualche gruppo camorristico facente capo alla nostra organizzazione ha rapporti all'estero.

PRESIDENTE. Con quali paesi?

PASQUALE GALASSO. Con l'Olanda.

PRESIDENTE. Con la Germania?

PASQUALE GALASSO. In Germania c'è già il gruppo Licciardi, Contini, Mallardo che, dopo la caduta del muro di Berlino, è presente a Berlino città, maggiormente con negozi di elettrodomestici, abbigliamento e un po' con i supermercati. Questo vuol dire che se domani voglio andare a Berlino non posso andare...

PRESIDENTE. Perché? Per rispetto delle zone?

PASQUALE GALASSO. Per rispetto verso Licciardi: loro hanno la precedenza assoluta, perché sono già presenti nella zona. Così, se vanno altri elementi camorristici che non sono graditi a Licciardi, devono cambiare zona. 
PRESIDENTE. Le risulta che siano presenti soltanto a Berlino, o anche in altri posti della Germania?

PASQUALE GALASSO. Anche in altri posti, ed in Romania, dove pure il nostro gruppo è presente.

PRESIDENTE. Come mai proprio in Romania?

PASQUALE GALASSO. E' presente un nostro affiliato che è da sempre in stretti e buoni rapporti con Alfieri, già dal periodo della dittatura rumena, per l'importazione di legnami. Hanno fatto poi diversi altri investimenti.

PRESIDENTE. Questo soggetto è ancora li adesso?

PASQUALE GALASSO. Sì.

PRESIDENTE. Come si chiama?

PASQUALE GALASSO. Nusco Mario.

PRESIDENTE. In altre città della Germania?

PASQUALE GALASSO. Diciamo che in Germania ci sono diversi gruppi camorristici, ma ultimamente chi fa da padrone è il gruppo Licciardi, Mallardo, Contini.

PRESIDENTE. In Francia?

PASQUALE GALASSO. In Francia ci sono da sempre rapporti camorristici francesiCosa nostra; principalmente c'è il gruppo di Michele Zaza.

PRESIDENTE. Nella Francia del sud? 
PASQUALE GALASSO. Anche a Parigi.

PRESIDENTE. Per quale tipo di affari?

PASQUALE GALASSO. Tutti i diversi affari, almeno per quanto mi risulta: una volta, c'era principalmente il contrabbando di sigarette e negli ultimi anni sono presenti un po' in tutti gli altri affari, come possono essere il gioco d'azzardo, il racket, l'usura eccetera.

PRESIDENTE. E in Spagna?

PASQUALE GALASSO. Per quanto mi risulta, in Spagna e in Portogallo c'è stato Bardellino mentre oggi ci sono i Casalesi. In questi paesi Bardellino aveva cointeressenze, aveva creato un impero.

PRESIDENTE. Dove?

PASQUALE GALASSO. A Barcellona, Madrid e in altre località spagnole e portoghesi. Oggi sono presenti i Casalesi.

PRESIDENTE. E come si procura le armi la camorra? Lei ha fatto cenno ai rapporti con la 'ndrangheta; solo così o anche per altri canali?

PASQUALE GALASSO. Anche per altri canali, talvolta tramite elementi mafiosi e dall'estero.

PRESIDENTE. Da quali paesi?

PASQUALE GALASSO. Dalla Germania, Francia e Olanda.

PRESIDENTE. Anche dall'Olanda? 
PASQUALE GALASSO. Sì.

PRESIDENTE. A Santo Domingo ci sono vostre presenze?

PASQUALE GALASSO. Dal 1984 in poi a Santo Domingo è presente Bardellino.

PRESIDENTE. Racconti alla Commissione.

PASQUALE GALASSO. Nel 1984 andai a trascorrere una vacanza a Santo Domingo e vidi una tranquillità assoluta, anche se lì vi sono quei problemi sociali che ci sono. Al ritorno mi incontrai con Bardellino.

PRESIDENTE. Con quale Bardellino?

PASQUALE GALASSO. Antonio. Gli parlai di questa tranquillità assoluta, così come ne parlai anche con Antonio Malvento il quale, dopo che gliene parlai, insieme con Antonio Ammaturo, fratello di Umberto, si recò a Santo Domingo dove cominciarono a fare vari investimenti e ad occupare quella zona. Una volta venuto a conoscenza di questa occupazione, Bardellino, il quale non stimava tanto Malvento e Ammaturo, andò a Santo Domingo, li espropriò di tutti i loro beni, comprese le abitazioni, li cacciò via e da quel momento è stato presente il gruppo di Bardellino.

PRESIDENTE. Bardellino ha cacciato gli altri?

PASQUALE GALASSO. Fino alla sua morte Bardellino è rimasto a Santo Domingo. Il giorno prima che scomparisse è partito da Santo Domingo per arrivare in Brasile e lì lo ammazzarono.

PRESIDENTE. In Brasile?

PASQUALE GALASSO. Sì. 
PRESIDENTE. Insieme con chi andò a Santo Domingo?

PASQUALE GALASSO. Andai io, l'ultimo sindaco di Poggiomarino, fino a quando non è stato sciolto il comune.

PRESIDENTE. Chi era?

PASQUALE GALASSO. Salvatore Lettieri, che fa capo a Pomicino; c'erano anche un altro consigliere comunale di Poggiomarino, Parisi e un mio nipote.

PRESIDENTE. Come mai andaste a fare quel giro?

PASQUALE GALASSO. Salvatore Lettieri aveva un amico, che conoscevo anch'io, e ne approfittammo per andare lì. Salvatore Lettieri, però, mi presentò un gruppo di ingegneri svizzeri i quali mi prospettarono un investimento su Santo Domingo per costruire un grande residence. Dopo aver chiarito che nel caso non avrei comprato soltanto un lotto ma che ero interessato all'affare, concordammo di andare lì per vedere da vicino questa zona ed eventualmente entrare in società per questa speculazione immobiliare.

PRESIDENTE. Con gli svizzeri o con Lettieri?

PASQUALE GALASSO. Con gli svizzeri e con Lettieri.

PRESIDENTE. Anche con l'altro consigliere comunale?

PASQUALE GALASSO. Sì.

PRESIDENTE. Quindi non fu soltanto una vacanza!

PASQUALE GALASSO. No, è stata l'uno e l'altro. 
PRESIDENTE. Come è finito questo investimento?

PASQUALE GALASSO. Finì che ritornai da Santo Domingo, ebbi l'ennesimo problema giudiziario e mi allontanai da questo investimento immobiliare ma feci presente alla mia organizzazione, a Carmine Alfieri, Antonio Malvento e poi a Bardellino della tranquillità di quella zona dove si poteva investire.

PRESIDENTE. Bardellino poi andò e investì lui?

PASQUALE GALASSO. Prima Malvento e Ammaturo poi ci andò Bardellino e, dato che non sopportava né Ammaturo né Malvento, li espropriò totalmente dei loro investimenti prendendosi anche le loro abitazioni e li cacciò via.

PRESIDENTE. Li comprò e li cacciò in malo modo?

PASQUALE GALASSO. In malo modo, visto che giustamente era un latitante di un certo livello e aveva bisogno di quelle zone, doveva stare tranquillo e non poteva essere disturbato.

PRESIDENTE. Quelli cedettero tutto e se ne andarono tranquillamente?

PASQUALE GALASSO. Sì, perché conoscevano bene Bardellino e si conoscevano loro stessi che sono stati speculatori e pessimi elementi malavitosi napoletani.

PRESIDENTE. Non avrebbero resistito a Bardellino?

PASQUALE GALASSO. Bardellino conosceva i Malvento di razza, di famiglia, pure imparentati con gli Alfieri, ma sapeva tutti i loro difetti.

PRESIDENTE. Perché lei ha sempre respinto le offerte di Cutolo? 
PASQUALE GALASSO. Ho visto Cutolo sempre come un delinquente nato; dico questo per dire di uno che fa il delinquente dal mattino alla sera, che pratica quei modi antisociali, asociali, quei modi di approfittare del miserabile, dell'ignorante per scopi suoi. Non l'ho mai apprezzato come uomo; è uno sceneggiatore, l'ho ritenuto sempre pericoloso.

PRESIDENTE. Cosa vuol dire "è uno sceneggiatore", uno che finge?

PASQUALE GALASSO. No, sceneggiatore, intrigante, ambiguo, traditore, un uomo che si serve dell'ignorante e eventualmente poi lo ammazza. Questi sentimenti, almeno in me e in qualche altro elemento mio amico... Abbiamo conosciuto Cutolo fin dal 1975-76 e lo definimmo una pessima figura.

PRESIDENTE. Cutolo ha ordinato in carcere omicidi, oltre quello di don Mico Tripodo?

PASQUALE GALASSO. Sì, ha fatto ammazzare colui che ha creato Cutolo, la sua struttura, quello che ha contribuito alla creazione della NCO, Antonino Cuomo, che nel 1975-76 per diversi mesi è stato nella mia stessa cella. Era un ragazzo nato per strada, Cutolo lo ha levato da lì, gli ha dato qualche migliaio di lire e quindi ha sfruttato il coraggio di questo ragazzo e il suo carisma verso gli altri carcerati. Questo giovane riuscì a fuggire dal carcere di Santa Maria Capua Vetere e dopo qualche mese fece evadere Cutolo dal carcere. Antonino Cuomo si accorse che Cutolo era una pessima figura e mi risulta che nel carcere speciale di Poggioreale Antonino Cuomo sfidava ogni giorno Cutolo, facendo il passeggio, e dandogli dei chiarimenti, perfino il chiarimento sull'arresto di Antonino Cuomo.

PRESIDENTE. Sul suo stesso arresto?

PASQUALE GALASSO. Antonino Cuomo diceva che era stato arrestato, che l'aveva fatto arrestare Cutolo per non far arrestare la sorella. 
PRESIDENTE. Rosetta?

PASQUALE GALASSO. Antonino Cuomo diceva che Cutolo se lo era venduto in cambio di Rosetta Cutolo (siamo nel 1979-80 e c'erano delle indagini).

PRESIDENTE. Cutolo era destinato alla polizia o ai carabinieri dove c'era Antonino Cuomo, avendo in cambio la salvaguardia della sorella? E' così?

PASQUALE GALASSO. Sì, tant'è vero che Cutolo, nonostante tutto quello che Antonino Cuomo gli ha fatto, lo fece ammazzare nel carcere di Poggioreale. Ma questo non basta: dopo pochi giorni fece ammazzare sulla strada che va da Sant'Antimo a Castel Capuano la moglie di Antonino Cuomo che doveva andare a testimoniare dai giudici a Napoli. Era una giovane ragazza che aveva in braccio il figlio di pochi mesi di Antonino Cuomo. E poi tante altre cose. Vorrei parlare di Cutolo. Non so se voi vi siate resi conto della ferocia di Cutolo, particolarmente come soggetto che si pone contro lo Stato, che rappresenta l'antistato. In sintensi, dopo il sequestro Cirillo, che rappresenta una data importante...

PRESIDENTE. Poi ci arriviamo...

PASQUALE GALASSO. Ad un certo punto, Cutolo ha tutto l'apparato dello Stato al suo fianco, diciamo sotto la sua protezione e amicizia, e si permette di attaccare lo Stato frontalmente, tentando di ammazzare, oltre che i poliziotti, il direttore del carcere di Poggioreale, sette, otto o dieci guardie, carabinieri ed anche due magistrati. Questi ultimi non sono morti, altrimenti oggi li compiacevate, e uno di essi è Lamberti. Dopo il sequestro Cirillo, Cutolo ha attentato alla vita di Lamberti e di Gagliardi. Questo deve farvi capire la ferocia di Cutolo, che, se per un attimo fosse andato avanti il rapporto che aveva intessuto con una parte delle istituzioni e con certi politici, quella che è oggi la realtà di Palermo, in Campania l'avremmo avuta già dieci anni fa. Questo almeno in base alle mie riflessioni di quell'epoca, quando ho vissuto i fatti in prima persona, tanto che ho desiderato la morte per liberarmi, quando ogni mattina leggevo sui giornali quello che riusciva a fare Cutolo. A gennaio mi è morto mio 
fratello, e a gennaio o marzo Cutolo ammazzò un piccolo pregiudicato, solo perché non condivideva le sue idee, a Roccarainola, assieme al figlio di otto anni...

PRESIDENTE. Ammazzò padre e figlio?

PASQUALE GALASSO. Padre e figlio, un bambino di otto anni! Prendete i rapporti dell'epoca. Quando la mattina uno si sveglia e legge che è morta Simonetta Lamberti, figlia di un magistrato... Sono cose che fanno inorridire! Quando a settembre ho letto che ad Avellino era stato compiuto un attentato alla vita di Gagliardi, solo perché egli era già arrivato a ciò cui voi state arrivando oggi, cioè all'intreccio tra politica, affari, imprenditori e camorra... Voglio farvi capire la ferocia di Cutolo, il quale aveva sposato la mentalità mafiosa, cioè di andare contro lo Stato. Signor presidente, nella mia vita criminale, né io né Enzo Moccia ci siamo mai sognati di sparare addosso ad un carabiniere, ad un poliziotto. Ho rischiato mille volte di essere arrestato, ma ho abbandonato le armi per non farmi tentare di rispondere al fuoco.

PRESIDENTE. Perché in Campania non sono mai stati ammazzati magistrati ma alcuni poliziotti e molti carabinieri?

PASQUALE GALASSO. Questo si deve principalmente alla sconfitta di Cutolo, a quello che io le dicevo: se Cutolo andava avanti...

PRESIDENTE. Ammaturo è stato ammazzato da Cutolo?

PASQUALE GALASSO. Secondo me sì, da Cutolo con la complicità di apparati istituzionali. Per me è una cosa vergognosa, perché io quell'epoca l'ho vissuta in prima persona. Voglio dirle che se andava avanti questa mentalità di mafia di Cutolo e di Nuvoletta, Napoli sarebbe diventata uguale a Palermo. Non faccio riferimento a chiacchiere ma agli attentati che ci sono stati: Salvi, il direttore del carcere di Poggioreale, è morto solo perché probabilmente ha avuto un alterco con Cutolo. Si può mai condividere una cosa del genere? Sette, otto, dieci, quindici guardie carcerarie muoiono soltanto perché non fanno una cortesia a Cutolo o non portano 
armi a Poggioreale! Un maresciallo dei carabinieri muore dopo aver consegnato un'arma nel tribunale di Napoli!

PRESIDENTE. Questo maresciallo consegnava un'arma a Cutolo?

PASQUALE GALASSO. A un associato di Cutolo, che ammazzò un affiliato, diciamo un grosso pregiudicato di Napoli, e attentò alla vita di Gennaro Licciardi, a Castel Capuano...

PRESIDENTE. Quindi, in tribunale?

PASQUALE GALASSO. Sì, in tribunale, a Castel Capuano. Siamo nel 1982...

PRESIDENTE. Poi fu ucciso questo maresciallo?

PASQUALE GALASSO. Sì. Dovete sapere che Cutolo si serviva di queste persone e poi le ammazzava.

PRESIDENTE. Poi arriveremo alla vicenda Cutolo. Quando lei parla di apparati istituzionali dei quali si è avvalso Cutolo, a quali fa riferimento?

PASQUALE GALASSO. Dovete partire, comunque, dalla vicenda Cirillo, da tutti quegli apparati che vanno da Cutolo e tramano contro gli interessi dello Stato per la liberazione di Cirillo. Dovete partire da quegli appoggi...

PRESIDENTE. Allora, visto che ci siamo e che è inutile girarci attorno, racconti questa vicenda.

PASQUALE GALASSO. E' l'aprile 1981. Sequestrano Cirillo. Certi politici, principalmente la corrente dorotea, andavano in cerca di vari appoggi camorristici e delinquenziali per trovare la loro disponibilità affinché si salvasse la vita di Ciro Cirillo. 
PRESIDENTE. Per cortesia, faccia nomi e cognomi, così capiamo bene.

PASQUALE GALASSO. In tutto questo contesto, il professore Boccia, di Poggiomarino, all'epoca presidente della USL 33 della provincia di Napoli, referente di Gava, in ottimi rapporti con Gava, mi chiese se io e Alfieri potevamo intervenire, se potevamo dare una mano per la liberazione di Cirillo. Questo, oltre che per fare una cortesia lui a Gava, anche perché consentisse al professor Boccia di avere l'appoggio di Gava alle prossime elezioni, in quanto voleva candidarsi alla Camera. Io ne parlai con Alfieri ed egli mi disse: "Non ci immischiamo in questa cosa". Nel contempo sapemmo che Gava, in prima persona, elementi della corrente dorotea, altri onorevoli, in prima persona Patriarca (almeno all'epoca ci risultava) si erano messi in contatto con Cutolo nel carcere di Ascoli Piceno per trattare la liberazione di Cirillo. Questo avviene ed accresce in noi il timore, la paura che Cutolo potesse facilmente aggredirci.

PRESIDENTE. Perché diventava più forte?

PASQUALE GALASSO. Sì. Siamo in quell'anno dove Cutolo ancora non si permetteva, eventualmente, di scagliarsi in una guerra contro tutti i gruppi camorristici. Siamo nell'epoca in cui Nuvoletta e gli elementi di Cosa nostra mediavano, trattavano per una tregua, per una pace con Cutolo. Quindi, da questo accordo tra Cutolo, quei politici, i servizi segreti e tutti gli apparati dello Stato che hanno concordato la liberazione di Cirillo, Cutolo prende il potere assoluto. Secondo lui, Cutolo è il più forte. Quindi, ci aggredisce ammazzando Salvatore Alfieri, perché pensava che lui potesse tutto. Nella stessa epoca della morte di Salvatore Alfieri, Cutolo aggredisce mio fratello, i vari elementi che rappresentavano degnamente lo Stato, tra i quali Lamberti, Gagliardi, Ammaturo, Salvi e carabinieri e poliziotti. Ci dimostra la sua ferocia e la sua teoria mafiosa, secondo la quale se uno dà fastidio bisogna eliminarlo; è inutile che... non ci sono... E poi da tutto questo nasce la guerra nostra, insieme a Bardellino, contro Cutolo, fino alla sconfitta di questo. Diciamo, tutti quegli 
appoggi, tutti quei politici che si erano serviti sia Cutolo sia Nuvoletta passano nelle mani di Alfieri.

PRESIDENTE. Come passano?

PASQUALE GALASSO. Ritornano nelle mani di Alfieri, perché la loro mentalità è quella che a loro non interessa di avere rapporto, fedeltà con un camorrista, a loro interessa solamente il camorrista del momento, perché sanno prima o poi, sanno la mentalità, i politici, almeno questi politici, sanno la mentalità dei camorristi: oggi c'è un camorrista in una zona, domani non c'è più, quindi si associano all'altro camorrista, intrecciano rapporti con l'altro camorrista.

PRESIDENTE. Come si spiega il passaggio di questi uomini politici da Cutolo ad Alfieri?

PASQUALE GALASSO. Durante la guerra con Cutolo, nel 1982, noi eravamo aggiornati all'interno della nostra organizzazione e pure perché Cutolo in prima persona ce lo faceva sapere, lo mandava a dire. Eravamo a conoscenza e sapevamo di una certa tensione tra Cutolo e tutti quegli apparati dello Stato, compresi i politici, con a capo Gava, che non avevano mantenuti quei patti, quel patto o quei patti per la liberazione di Cirillo.

PRESIDENTE. E quali erano?

PASQUALE GALASSO. Noi sapevamo principalmente la liberazione dello stesso Cutolo e altri benefici che Cutolo aveva chiesto per la liberazione di Cirillo. Questi non si avverarono, non vennero mantenuti e Cutolo minacciava tutti questi apparati, un po' ricattava ma principalmente, esternamente, lo faceva Casillo, che nel frattempo si era trasferito a Roma, con una certa protezione, principalmente dei servizi segreti. Io sapevo con sicurezza, da parte di Cillari, mio infiltrato nei cutoliani, che Casillo girava per Roma, lui e tutto lo staff, il direttivo dei cutoliani, con una 
tessera dei servizi segreti e quindi lui... a Roma loro si sentivano sicuri, tant'è vero che Casillo veniva nel napoletano, faceva delle riunioni ma la sera ritornava a Roma.

PRESIDENTE. Era più sicuro a Roma che a Napoli.

PASQUALE GALASSO. Sì. Queste informazioni le so per certo perché me le trasferiva Pino Cillari che stava...

PRESIDENTE. Che era un suo infiltrato dentro al gruppo?

PASQUALE GALASSO. Sì, che poi è quello che mi dava...

PRESIDENTE. Come avviene poi...

PASQUALE GALASSO. Nell'epoca Cirillo, dopo terremoto, epoca dopo Cirillo, quei contatti per la liberazione di Cirillo, Cutolo trattò pure il controllo degli appalti pubblici che dovevano avvenire e già c'era una commistione, una simbiosi, un intreccio, un accordo tra i politici, imprenditori vicini a Cutolo e ai politici e lo stesso Cutolo; questo sia nella zona nostra, nel napoletano, sia anche nell'avellinese, che all'epoca Avellino ancora era una città non con problemi camorristici. Questo interessamento su Avellino di Cutolo e degli imprenditori facenti capo a Cutolo venne a capo del dottor Gagliardi, almeno a quello che a me mi risulta e mi consta in prima persona, tanto da far cominciare a fare delle indagini e perciò Cutolo subito pensò di ammazzarlo. Dopo l'omicidio Casillo.

PRESIDENTE. Ho capito. Spieghi bene l'omicidio Casillo, perché avviene. Cirillo è liberato a luglio del 1981, no?

PASQUALE GALASSO. Sì.

PRESIDENTE. Poi c'è Cutolo che a questo punto fa uccidere delle persone. 
PASQUALE GALASSO. Sì.

PRESIDENTE. Poi c'è l'omicidio di Casillo, cioè dell'uomo di Cutolo. Come avviene questo omicidio, chi lo decide e perché? Perché lei dice sempre "dopo l'omicidio Casillo, Alfieri diviene forte". Spieghi bene.

PASQUALE GALASSO. A gennaio 1982 mi muore mio fratello Nino.

PRESIDENTE. Nel gennaio?

PASQUALE GALASSO. Sì, nel gennaio del 1982. Dopo circa un mese...

PRESIDENTE. Che è ammazzato dai cutoliani?

PASQUALE GALASSO. Sì.

PRESIDENTE. Sempre in questa...

PASQUALE GALASSO. Sì. Mi trasferisco a Roma, compro una villa a Castelgandolfo. Io e tutti i miei familiari ci trasferiamo a Roma. Io all'epoca ero latitante, quindi questa villa mi serviva sia per me ma principalmente per proteggere i miei familiari. In quell'epoca, io incontrai Pino Cillari e poi ho conosciuto, tramite Pino Cillari, la moglie, l'attuale moglie, Matilde Ciarlante. Frequentavo Pino Cillari in quell'epoca. Pino Cillari un giorno mi disse che a Roma lui si era incontrato con Vincenzo Casillo e tutto lo staff cutoliano. Al che io invitai Pino Cillari ad una serenità nei miei riguardi e esplicitamente gli dissi: "Pino, non vorrei morire per le mani di questa gente. Se ciò mi accade, mi accadde perché tu mi vendi". Lui mi giurò fedeltà e, una volta avuto questo, io gli dissi apertamente la mia intenzione di ammazzare a Casillo e, diciamo, questo gruppo, che era responsabile della morte di mio fratello. Cillari mi promise fedeltà e in più mi disse: "Incominciamo a studiare come fare per individuare Casillo e tutti quanti". Cillari riuscì a mettersi in contatto con Casillo e gli altri e man mano mi incominciò a delucidare tutti gli appoggi di Casillo, gli appoggi delle persone su 
Roma, che erano i fratelli Rizzo (che avevano una tenuta sulla via Cassia e un negozio a Piazza di Spagna a Roma, trafficavano principalmente per le scommesse clandestine su Tor di Valle e altri traffici), c'erano i fratelli Casella di Salerno, Mario Casella, Doganieri e altri, c'era Rolando Tortora, convivente della madre dei fratelli Rizzo, la signora Di Maio. Quindi, mi localizzò un po' tutti i vari appoggi di Casillo, i suoi uomini di fiducia. Io in quell'epoca mi organizzai per attentarli.

PRESIDENTE. Parlò con Alfieri dell'attentato?

PASQUALE GALASSO. Sì, poi, subito - mi organizzai per attentarli, per ammazzarli ne parlai con Alfieri in una riunione, con Alfieri e gli altri miei amici, all'epoca erano pochissimi. Dopo una decisione... Alfieri era un po' incredulo che Pino Cillari era stato capace, era capace di individuarci Casillo, perché gli davamo la caccia in tutta la Campania. Ma insieme agli uomini fidati pure di Alfieri, ci portammo su Roma, contattammo Cillari, Cillari ci dimostrò la realtà di tutto. Appresa pure loro la fedeltà di Cillari nei riguardi miei e di Alfieri, ci incominciammo ad organizzare per attentare Casillo per la strada o nei suoi vari appoggi. Per circa 5, 6, 7 mesi ci è scappato in più di un attentato. Figuratevi, come in quell'epoca ero imbestialito, che io e il mio amico Enzo Moccia andammo a studiare a Piazza di Spagna a fare una strage là in mezzo, pur sapendo eventualmente che potevamo morire ammazzati, perché Pino Rizzo aveva questo negozio, questo appoggio, dove Casillo, mi diceva Cillari, era solito andare, lui con altri cinque, sei elementi. A Piazza di Spagna vicino a degli istituti bancari, quindi stavano guardie giurate e poi Piazza di Spagna è sempre piena di carabinieri, poliziotti. Mi ricordo che io e Enzo Moccia ci studiammo la via della fuga, ce ne andavamo per la metropolitana. Poi, fatto sta, andammo a fare questi appostamenti ma furono infruttuosi. Poi, alla fine, nell'anno 1982, estate o autunno, il capitano Niglio, il capitano della compagnia di Nocera Inferiore venne su Roma e arrestò Salvatore Di Maio che era scappato dal carcere di Salerno. Quindi, Casillo e gli altri suoi associati temettero che il capitano Niglio avesse scoperto tutti i loro appoggi e chiesero a Cillari dei nuovi appoggi, non quelli che loro avevano sfruttato fino a quel tempo. Cillari mi riferì tutto. Mi organizzai insieme a Cillari, dandogli pure dei soldi, 100-120 milioni di lire, per comprare un appartamento a 
Primavalle e un altro appartamento a via Boccea, dietro via Boccea, poco distante da Primavalle, affinché noi potessimo agire contemporaneamente sia su Casillo sia sugli altri affiliati. Questa storia, questo fatto, lo riferì ad Alfieri: siamo nell'estate 1982, quando i cutoliani, ancora una volta, tramite Nuvoletta e con i loro appoggi mafiosi, invitarono noi e Bardellino ad una tregua, ad una pace. Si riandò di nuovo a fare quelle riunioni dai Nuvoletta - siamo nell'estate 1982 - ma Bardellino fu molto esplicito nei confronti dei cutoliani nel dire...

PRESIDENTE. I cutoliani vennero lì?

PASQUALE GALASSO. Si fece di nuovo un'altra riunione come quella del 1981, promotore sempre il Nuvoletta, dove Bardellino e noi, principalmente, chiedemmo che a capotavola non si sedesse più Lorenzo Nuvoletta, proprio in virtù della sua personalità viscida, melmosa, ma alla fine si condivise...

PRESIDENTE. Chi volevate che si sedesse a capotavola?

PASQUALE GALASSO. Volevamo eventualmente qualche altro personaggio malavitoso campano, ma alla fine si condivise che al posto di Lorenzo Nuvoletta si sedesse un suo fratello: ma questo, credo, sempre per volontà del doppio gioco dei Nuvoletta con dietro i mafiosi. Bardellino e alla fine lo stesso Alfieri accettarono questa riunione, alla quale io non andai; in quella stessa giornata organizzammo di attentare Casillo e tutti i cutoliani ma ci sfuggirono. Non andai anche perché avevo giurato vendetta nei riguardi di Nuvoletta, in virtù della loro pessima personalità: lo sapevano Alfieri, pochi altri miei associati e dopo pure lo stesso Bardellino.

PRESIDENTE. La riunione, quindi, finisce con un niente di fatto?

PASQUALE GALASSO. Finisce così, con Bardellino che dice che non è possibile perché, per avere una pace con i cutoliani, lui pretende che muoiano i responsabili delle morti dei nostri cari. Quindi, non se ne fece niente. 
PRESIDENTE. La condizione era che morissero quelli che avevano assassinato i vostri cari?

PASQUALE GALASSO. Sì.

PRESIDENTE. E quelli non erano d'accordo?

PASQUALE GALASSO. Per forza maggiore: erano responsabili Casillo, Cuomo, Giappone, diciamo un po' i capi e la dirigenza di Cutolo. Non se ne fece niente e ritornando a Roma... In quell'epoca, nel 1982, Alfieri già veniva fuori come uno dei nuovi capi camorristici campani; Cutolo già stava in difficoltà nonostante che minacciasse e ricattasse tutti gli apparati dello Stato. Era quella l'epoca...

PRESIDENTE. Lei come fa a sapere che ricattava gli apparati dello Stato?

PASQUALE GALASSO. Diciamo che principalmente era lo stesso Cutolo che lo spifferava e lo diceva, ma pure perché nel frattempo noi avevamo già sequestrato qualche elemento cutoliano, avevamo ammazzato qualche persona, sequestrandola ed interrogandola. Questo lo facevamo noi come, d'altro canto, Bardellino.

PRESIDENTE. Quindi sapevate le notizie in questo modo?

PASQUALE GALASSO. Sì; ne avevamo noi e ci arrivavano da Bardellino; inoltre, i fatti lo dimostravano per i vari attentati mortali che Cutolo faceva. Ricordo l'omicidio del commissario Ammaturo, mi sembra vice questore, di cui nel nostro gruppo, all'epoca, parlavamo un po', perché, per quanto mi risulta, l'Ammaturo non aveva fatto nulla di grave per meritarsi quella morte, che era per noi inspiegabile. Quindi, quello era un messaggio importante che Cutolo mandava a noi altri, per mostrare che non aveva limiti e frontiere. Come questo, posso dire di tanti altri casi. Quella era l'epoca in cui Cutolo ricattava anche questi politici e in cui Alfieri - forse già da quel momento - aveva cominciato a riprendere i contatti politici che aveva una volta, principalmente con il senatore Patriarca... 
PRESIDENTE. Contatti che si erano interrotti?

PASQUALE GALASSO. Contatti che si erano interrotti durante la vicenda Cirillo, in quel paio di anni.

PRESIDENTE. Come fa a riprendere i contatti?

PASQUALE GALASSO. Credo principalmente attraverso sue persone e poi con i referenti della corrente dorotea nella zona nolana-vesuviana. Pensate che Alfieri conosce Ciccio Catapano, un fedelissimo della corrente dorotea, particolarmente di Gava, sin dagli anni settanta, quando aveva un mobilificio sotto l'abitazione di Ciccio Catapano; conosce Meo già da diversi anni; conosce Riccio che è nato malavitosamente (Riccio e il fratello hanno pure dei precedenti penali) e che ultimamente doveva candidarsi alla Camera; conosce gli altri referenti politici della corrente dorotea. Già in quell'epoca, quindi, Alfieri riprende questi rapporti, perché Cutolo aveva già subìto diverse morti e già non era presente nella nostra zona, dopo cinque o sei mesi di guerra cruenta.

PRESIDENTE. Quindi, vi è questa guerra, Cutolo si indebolisce, Alfieri riprende i suoi rapporti: torniamo ora a Casillo.

PASQUALE GALASSO. Voglio aggiungere che per quell'epoca, con un po' di attenzione, voi potreste capire come anche lo Stato - parlo principalmente delle forze dell'ordine - era impreparato ad una ferocia così inaudita: chi mai avrebbe pensato che nel 1982 ci sarebbero stati cinque-seicento morti, come ci sono stati? Vi siete mai spiegati come mai all'improvviso capiti tutto questo? La spiegazione più importante è il caso Cirillo ed il dopo terremoto, gli appalti, l'affare: altrimenti, una guerra così cruenta non può succedere, se non c'è a monte un accordo tra referenti politici, camorra e imprenditori. Voglio portarvi ad una riflessione chiara, elementare scusatemi: non è possibile che vi siano 1.500 morti in dieci anni, che nel solo 1982, dopo il caso Cirillo, vi siano 500 morti, se a monte non c'è un'intesa, una 
simbiosi, una commistione, un accordo fra parti che devono dividersi la torta del dopo terremoto. Cutolo non può uscire pazzo, ammazzare, aggredire 50-100 gruppi malavitosi, se non c'è una finalità, uno scopo: lo scopo è l'arricchimento. Il politico non può scendere a patti con il camorrista se alla fine non ha l'interesse suo: non è l'interesse dei partiti, almeno secondo me. Io l'ho toccato con mano: capisco che per voi sia difficile comprendermi, ma riflettete, approfondite questo fenomeno [...] 